PREPARED FOR THE U.S. DEPARTMENT OF ENERGY, UNDER CONTRACT DE-AC02-76CH03073

PPPL-3930

PPPL-3930

UC-70

Statistical Plasma Physics in a Strong Magnetic Field:

Paradigms and Problems

by

J.A. Krommes

March 2004

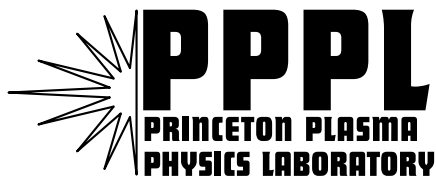

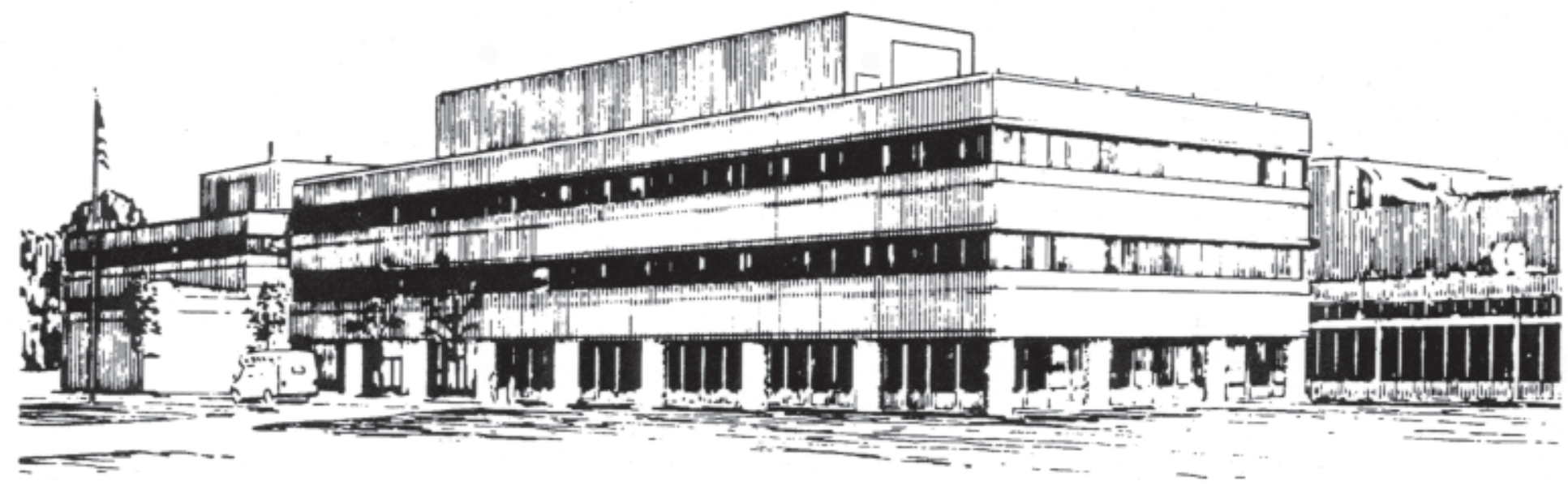

PRINCETON PLASMA PHYSICS LABORATORY PRINCETON UNIVERSITY, PRINCETON, NEW JERSEY 


\section{PPPL Reports Disclaimer}

This report was prepared as an account of work sponsored by an agency of the United States Government. Neither the United States Government nor any agency thereof, nor any of their employees, makes any warranty, express or implied, or assumes any legal liability or responsibility for the accuracy, completeness, or usefulness of any information, apparatus, product, or process disclosed, or represents that its use would not infringe privately owned rights. Reference herein to any specific commercial product, process, or service by trade name, trademark, manufacturer, or otherwise, does not necessarily constitute or imply its endorsement, recommendation, or favoring by the United States Government or any agency thereof. The views and opinions of authors expressed herein do not necessarily state or reflect those of the United States Government or any agency thereof.

\section{Availability}

This report is posted on the U.S. Department of Energy's Princeton Plasma Physics Laboratory Publications and Reports web site in Fiscal Year 2004. The home page for PPPL Reports and Publications is: http://www.pppl.gov/pub_report/

DOE and DOE Contractors can obtain copies of this report from:

U.S. Department of Energy

Office of Scientific and Technical Information

DOE Technical Information Services (DTIS)

P.O. Box 62

Oak Ridge, TN 37831

Telephone: (865) 576-8401

Fax: (865) 576-5728

Email: reports@adonis.osti.gov

This report is available to the general public from:

National Technical Information Service

U.S. Department of Commerce

5285 Port Royal Road

Springfield, VA 22161

Telephone: $1-800-553-6847$ or

(703) $605-6000$

Fax: (703) 321-8547

Internet: http://www.ntis.gov/ordering.htm 


\title{
Statistical Plasma Physics in a Strong Magnetic Field: Paradigms and Problems
}

\author{
J. A. Krommes* \\ Plasma Physics Laboratory, Princeton University, \\ P.O. Box 451, Princeton, New Jersey 08543
}

(Dated: March 2, 2004)

\begin{abstract}
An overview is given of certain aspects of fundamental statistical theories as applied to strongly magnetized plasmas. Emphasis is given to the gyrokinetic formalism, the historical development of realizable Markovian closures, and recent results in the statistical theory of turbulent generation of long-wavelength flows that generalize and provide further physical insight to classic calculations of eddy viscosity. A Hamiltonian formulation of turbulent flow generation is described and argued to be very useful.
\end{abstract}

PACS numbers: 52.35.Ra, 47.27.Gs

*Electronic address: krommes@princeton.edu 


\section{INTRODUCTION}

The purpose of this paper is to describe the current state of affairs regarding certain topics in fundamental statistical plasma physics, with emphasis on techniques and recent results especially relevant for strongly magnetized plasmas. Such plasmas are particularly useful in fusion research; however, no detailed knowledge of fusion physics is required or used in the discussion.

In Sec. 2 I provide some introductory plasma-physics background on the gyrokinetic formalism used in modern discussions of low-frequency fluctuations in magnetized plasmas. This is a reduced kinetic (velocity-space) description that efficiently replaces the more comprehensive Vlasov equation when only low-frequency, long-wavelength fluctuations are of concern. Moments of the gyrokinetic equation lead to nonlinear gyrofluid equations that are the plasma analogs of the Navier-Stokes equation. Several important equations are briefly derived or cited, including the Hasegawa-Mima equation (directly analogous to the Charney equation for Rossby waves) and the Hasegawa-Wakatani equations.

In Sec. 3 I mention some past and recent plasma applications of statistical methods, including calculations of gyrokinetic noise and the development of realizable Markovian closures appropriate for problems with linear waves (ubiquitous in plasma physics as well as in geophysics and elsewhere).

In Sec. 4 I discuss in detail the statistical description of convective cells, including zonal flows (those will be defined later). This problem is closely related to calculations of eddy viscosity for Navier-Stokes fluids; I will note a precise and quantitative connection. The methodology unifies a number of interesting technical specialities, including statistical closure theory, the theory of weakly inhomogeneous spectral evolution equations, fieldtheoretic techniques, and the Hamiltonian formulation of nonlinear partial differential equations (PDE's).

The paper ends in Sec. 5 with some discussion and suggestions for future work. 


\section{INTRODUCTORY PLASMA-PHYSICS BACKGROUND, PARTICULARLY GYROKINETICS}

At the level of one-particle probability density functions (PDF's), the most fundamental description of a magnetized, collisional plasma is provided by the kinetic equation

$$
\begin{aligned}
\partial_{t} f_{s}(\boldsymbol{x}, \boldsymbol{v}, t)+\boldsymbol{v} \cdot \nabla f_{s} & +(q / m)_{s}\left(\boldsymbol{E}+c^{-1} \boldsymbol{v} \times \boldsymbol{B}\right) \cdot \partial_{\boldsymbol{v}} f_{s} \\
& =-C_{s}[f]
\end{aligned}
$$

where the collision operator $C$ is the Balescu-Lenard operator or, more practically, the Landau operator. Here $s$ is a species label, $q$ is the charge, $m$ is the mass, $\boldsymbol{E}$ is the electric field, $\boldsymbol{B}$ is the magnetic field, and square brackets denote functional dependence. The fields are determined from Maxwell's equations. Equation (1), which lives in the six-dimensional (6D) phase space of a generic particle (sometimes called the $\mu$ space), describes all particle motions, including the rapid gyrospiraling around the magnetic field at frequency $\omega_{c} \doteq q B / m c$, as well as both high- and low-frequency collective fluctuations. Its very completeness, however, poses substantial problems for both analysis and numerical simulation. For toroidal plasma systems (Fig. 1), which are routinely confined long enough to have particle distributions very close to a local Maxwellian, simple arguments [1] show that the most important microinstabilities are of low frequency, characteristically $\omega \sim \omega_{*}$, where $\omega_{*}$ is the so-called diamagnetic drift frequency inversely proportional to the scale length $L_{n}$ of the background density profile. Specifically, if $\boldsymbol{B} \propto \widehat{\boldsymbol{z}}$ and $\boldsymbol{\nabla}\langle n\rangle \propto-\widehat{\boldsymbol{x}}$, then $\omega_{*}=k_{y} V_{*}$, where

$$
V_{*} \doteq c T_{e} / e B L_{n}=\left(\rho_{\mathrm{s}} / L_{n}\right) c_{\mathrm{s}}
$$

the sound speed is $c_{\mathrm{s}} \doteq Z T_{e} / m_{i}\left(T_{e}\right.$ is the electron temperature and $Z$ is the atomic number of the ions), and $\rho_{\mathrm{s}} \doteq c_{\mathrm{s}} / \omega_{c i}$. One has

$$
\omega_{*} / \omega_{c i}=\left(k_{y} \rho_{\mathrm{s}}\right)\left(\rho_{\mathrm{s}} / L_{n}\right) \ll 1
$$

since typically $k_{y} \rho_{\mathrm{s}}=O(1)$ and $\rho_{\mathrm{s}} \ll L_{n}$. The disparity between $\omega_{*}$ and $\omega_{c i}$ implies that simulations of the complete dynamics are prohibitively lengthy by orders of magnitude, even with modern supercomputers.

The advent of the nonlinear gyrokinetic formalism was a major advance. Gyrokinetics is the statistical description of the motion of appropriately defined gyrocenters. Most 
heuristically, the motion of a gyrocenter is defined by the well-known particle drift velocities [2] such as the $\boldsymbol{E} \times \boldsymbol{B}, \nabla B$, and curvature drifts. However, there are subtleties, especially surrounding the role of the polarization drift $\boldsymbol{V}^{\text {pol }}=\omega_{c}^{-1} \partial_{t}\left(c \boldsymbol{E}_{\perp} / B\right)(\perp$ means perpendicular to $\boldsymbol{B}$ ), that can only be addressed with the aid of a systematic formalism. Following earlier attempts at linear gyrokinetics [3, 4], Frieman and Chen [5] published an important paper discussing the nonlinear problem. That work, however, divided the distribution function into separate parts for the background and the fluctuations, so the resulting gyrokinetic equation was not immediately useful for numerical simulation and also disguised certain important conservation properties of the dynamics. Lee [6] attempted to correct those deficiencies; however, his recursive procedure was soon superceded by the noncanonical Hamiltonian formalism of Dubin et al. [7] based on the Darboux techniques of Littlejohn [8]. After the later advent of even more efficient techniques based on differential one-forms [9], the formalism was further refined by Hahm [10]. For the most modern results, see Refs. 11 and 12 .

The Hamiltonian methods proceed by constructing a perturbative change of variables from an initial set of guiding-center variables appropriate for a static, spatially constant magnetic field. The goal of the transformation is to remove dependence on the gyro-angle $\theta$ from all components of the fundamental differential one-form [9]; when that is done, the magnetic moment $\mu$ is (adiabatically [13]) conserved. The procedure thus ensures both that the gyrocenter PDF is independent of $\theta$ (so the phase space is $5 \mathrm{D}$ rather than $6 \mathrm{D}$ ) and that the left-hand side of the kinetic equation contains no derivative with respect to $\mu$. These are considerable simplifications. For the simplest case of a constant magnetic field, the gyrokinetic equation reads (in the absence of collisions)

$$
\begin{aligned}
& \partial_{t} F_{s}\left(\boldsymbol{X}, \mu, v_{\|}, t\right)+v_{\|} \nabla_{\|} F_{s} \\
& \quad+\overline{\boldsymbol{V}}_{E} \cdot \nabla F_{s}+(q / m)_{s} \bar{E}_{\|} \partial_{v_{\|}} F_{s}=0 .
\end{aligned}
$$

Here $F$ is the PDF of gyrocenters and the overlines signify the effective (gyro-averaged) field at the position $\boldsymbol{X}$ of the gyrocenter [in $\boldsymbol{k}$ space, the averaging introduces the factor $\left.J_{0}\left(k_{\perp} v_{\perp} / \omega_{c i}\right)\right]$. Notably, only the $\boldsymbol{E} \times \boldsymbol{B}$ drift enters in the gyrokinetic equation; the polarization drift does not appear. Instead, the effects of polarization enter through the Poisson equation. That equation holds at the position $\boldsymbol{x}$ of the actual particles; it is 
$\nabla^{2} \phi(\boldsymbol{x}, t)=-4 \pi \rho(\boldsymbol{x}, t)$, where $\rho=\sum_{s}(\bar{n} q)_{s} \int d \boldsymbol{v} f_{x}(\boldsymbol{x}, \boldsymbol{v}, t)$ is the charge density. ${ }^{1}$ In this last expression, both the coordinates and the distribution function must be transformed in order to calculate $\rho$ from the gyrocenter distribution $F$. The polarization drift is contained in that change of coordinates $[7,13]$, the several steps of which are reviewed in App. C of Ref. 14. For $T_{i} \rightarrow 0$, the final result for a quasineutral plasma consisting of electrons and a single ion species is

$$
\lambda_{D e}^{2} \nabla^{2} \varphi+\rho_{\mathrm{s}}^{2} \nabla_{\perp}^{2} \varphi=-\left(\delta N_{i} / \bar{n}_{i}-\delta n_{e} / \bar{n}_{e}\right)
$$

where $\lambda_{D e}$ is the electron Debye wavelength $\left[\lambda_{D e} \doteq\left(T_{e} / 4 \pi \bar{n}_{e} e^{2}\right)^{1 / 2}\right]$ and $\varphi \doteq e \phi / T_{e}$. The $\rho_{\mathrm{s}}^{2}$ term describes the ion polarization density $n_{i}^{\text {pol }}$ (electron polarization is negligible); that is, $n_{i}=N_{i}+n_{i}^{\text {pol }}$, where $n_{i}^{\text {pol }}$ obeys $\partial_{t} n_{i}^{\text {pol }}+\nabla \cdot \boldsymbol{j}_{i}^{\text {pol }}=0$ with $\boldsymbol{j}_{i}^{\text {pol }} \doteq(\bar{n} q)_{i} \boldsymbol{V}_{i}^{\text {pol }}$. The quantity $\epsilon_{\perp} \doteq 1+\rho_{\mathrm{s}}^{2} / \lambda_{D e}^{2}=1+\omega_{p i}^{2} / \omega_{c i}^{2}\left[\omega_{p i} \doteq\left(4 \pi \bar{n}_{i} q_{i}^{2} / m_{i}\right)^{1 / 2}\right]$ defines what can be called the dielectric constant of the gyrokinetic vacuum [15]. The gyrokinetic regime is defined [16] by $\epsilon_{\perp} \gg 1$, which is frequently the case. ${ }^{2}$ Then, since usually $k_{\|} \ll k_{\perp}$, the $\lambda_{D e}^{2}$ term, which describes the deviation from absolute charge neutrality, is negligible. Upon scaling lengths to $\rho_{\mathrm{s}}$, one obtains the gyrokinetic Poisson equation

$$
\nabla_{\perp}^{2} \varphi=-\left(\delta N_{i} / \bar{n}_{i}-\delta n_{e} / \bar{n}_{e}\right)
$$

which describes quasineutrality within the gyrokinetic framework. It is quite convenient numerically, as one does not need to somehow enforce $\rho_{i}=\rho_{e}$ implicitly but instead can merely calculate the polarization density (or resulting potential) from the given imbalance of gyrocenter densities that exists at time $t$.

A simple physical interpretation can be given of the important $\rho_{\mathrm{S}}^{2} \nabla_{\perp}^{2} \varphi$ contribution to the gyrokinetic Poisson equation. Consider the $z$-directed vorticity $\Omega$ associated with the $\boldsymbol{E} \times \boldsymbol{B}$ motion: $\Omega \doteq \widehat{\boldsymbol{z}} \cdot \boldsymbol{\nabla} \times \boldsymbol{V}_{E}$. One readily finds that

$$
\Omega / \omega_{c i}=\rho_{\mathrm{s}}^{2} \nabla_{\perp}^{2} \varphi
$$

that is, for cold ions the ion polarization density is just the vorticity in appropriate units. This important property of strongly magnetized plasma is illustrated in Fig. 2, where it is

\footnotetext{
${ }^{1}$ The mean density $\bar{n}$ enters because of the convenient normalization convention $V^{-1} \int d \boldsymbol{x} \int d \boldsymbol{v} f=1$, where $V$ is the volume.

${ }^{2}$ The guiding-center limit is $\omega_{p i}^{2} / \omega_{c i}^{2} \rightarrow 0\left(\epsilon_{\perp} \rightarrow 1\right)$.
} 
shown that a deficiency of ion gyrocenters leads to a positive vorticity. It is thus expected that the dynamics of vorticity will play a crucial role in virtually all gyrokinetic calculations.

Integration of Eq. (4) over velocity leads to the continuity equation for gyrocenters. If ion parallel motion is ignored (because the ion inertia is very large) and for simplicity one again considers $T_{i} \rightarrow 0$, one obtains

$$
\partial_{t} N_{i}+\nabla \cdot\left(\boldsymbol{V}_{E} N_{i}\right)=0
$$

Let us divide $N_{i}$ into a background (mean-profile) part $\left\langle N_{i}\right\rangle$ and a fluctuating part $\delta N_{i}$ : $N_{i}=\left\langle N_{i}\right\rangle+\delta N_{i}$. The mean profile obeys

$$
\partial_{t}\left\langle N_{i}\right\rangle+\nabla \cdot \Gamma_{i}=0
$$

where $\boldsymbol{\Gamma}_{i} \doteq\left\langle\delta \boldsymbol{V}_{E} \delta N_{i}\right\rangle$, and the fluctuations obey

$$
\frac{\partial}{\partial t}\left(\frac{\delta N_{i}}{\bar{n}_{i}}\right)+V_{*} \frac{\partial \varphi}{\partial y}+\boldsymbol{\nabla} \cdot\left[\boldsymbol{V}_{E}\left(\frac{\delta N_{i}}{\bar{n}_{i}}\right)-\bar{n}_{i}^{-1} \boldsymbol{\Gamma}\right]=0 .
$$

Here the so-called "diamagnetic" term proportional to $V_{*}$ is a rewriting of $\boldsymbol{V}_{E} \cdot \boldsymbol{\nabla} \ln \left\langle N_{i}\right\rangle$; it thus describes advection of the background density gradient, not literally ion diamagnetism (which vanishes for $T_{i}=0$ ). For homogeneous statistics, the terms in $\boldsymbol{\nabla} \cdot \boldsymbol{\Gamma}$ vanish.

The gyrocenter continuity equation contains both $\delta N_{i}$ and $\delta \varphi$. The gyrokinetic Poisson equation relates those quantities, but also introduces the electron density as a new unknown. The classic approximation of Hasegawa and Mima (HM) [17] is to assume that the electron response is adiabatic: $\delta n_{e} / \bar{n}_{e}=\delta \varphi$ (the first-order part of a Maxwell-Boltzmann distribution). Poisson's equation then becomes

$$
\delta N_{i} / \bar{n}_{i}=\delta \varphi-\nabla_{\perp}^{2} \delta \varphi .
$$

Finally, substitution of this result into Eq. (10) leads to the Hasegawa-Mima equation (HME)

$$
\left(1-\nabla_{\perp}^{2}\right) \partial_{t} \delta \varphi+V_{*} \partial_{y} \delta \varphi+\boldsymbol{V}_{E} \cdot \nabla\left(-\nabla_{\perp}^{2} \delta \varphi\right)=0
$$

Note the appearance of the vorticity $\Omega=\nabla_{\perp}^{2} \varphi$ in several terms. The original derivation of HM from the Braginskii equations [18] (the fluid equations in particle coordinates) was not as physically transparent. The Hasegawa-Mima equation is the simplest paradigm for the nonlinear dynamics of drift waves. If one omits the two terms related to explicitly plasma 
effects [the term in $\partial_{t} \varphi$ (adiabatic electron response) and the term proportional to $V_{*}$ (effect of the background density gradient)], one is left with the 2D Navier-Stokes equation (NSE)

$$
\partial_{t} \Omega+\boldsymbol{V}_{E} \cdot \nabla \Omega=0
$$

The implicit assumption of HM was that the parallel wave number $k_{\|}$does not vanish. If it does, adiabatic electron response is no longer appropriate because there is no parallel potential modulation for the mobile electrons to neutralize. Because electron polarization is negligible, a frequently used approximation is to ignore electron response altogether for the $k_{\|}=0$ modes (convective cells). ${ }^{3}$ What results is the generalized HME

$$
\left(\alpha-\nabla_{\perp}^{2}\right) \partial_{t} \varphi+\alpha V_{*} \partial_{y} \varphi+\boldsymbol{V}_{E} \cdot \nabla\left(\alpha-\nabla_{\perp}^{2}\right) \varphi=0
$$

where

$$
\alpha \doteq \begin{cases}1 & k_{\|} \neq 0 \\ 0 & k_{\|}=0\end{cases}
$$

can be interpreted as a projection operator onto the DW subspace. For the CC's, one obtains precisely the 2D NSE.

Let us call the $k_{\|} \neq 0$ modes the drift waves (DW's). For the DW's, the linear dispersion relation is

$$
\omega=\frac{\omega_{*}}{1+k_{\perp}^{2}} .
$$

In this approximation there is no growth or dissipation. This is a nonphysical consequence of the neglect of Landau damping (the physically important wave-particle resonance, which is lost in fluid truncations that involve neglect of velocity cumulants beyond some order ${ }^{4}$ ) and collisions. Both of those effects introduce nonadiabatic electron response. If only collisions are taken into account (through the electron momentum equation), the system of equations due to Hasegawa and Wakatani (HW) [20] results:

$$
\begin{aligned}
\partial_{t} \Omega+\boldsymbol{V}_{E} \cdot \nabla \Omega & =\widehat{\delta}(\varphi-n)+\mu \nabla_{\perp}^{2} \Omega \\
\partial_{t} n+\boldsymbol{V}_{E} \cdot \nabla n & =\widehat{\delta}(\varphi-n)-\kappa \partial_{y} \varphi+D \nabla_{\perp}^{2} n
\end{aligned}
$$

\footnotetext{
${ }^{3}$ A subtle issue regarding modes with $k_{y}=0$ (zonal flows) and $k_{y} \neq 0$, especially in the presence of magnetic shear, cannot be discussed here.

4 More sophisticated fluid closures can model the Landau damping; see Ref. 19.
} 
where $n \equiv n_{e}, \kappa \doteq L_{n}^{-1}, \widehat{\delta} \doteq-D_{\|}^{-1} \nabla_{\|}^{2}$, and the equations are written for fluctuations only. The dissipative terms involving $\mu$ and $D$ can be added heuristically or can be derived from more detailed considerations. The virtue of this system is that it is both forced (there is a linear instability for some wavevectors [21] and dissipative, so it can achieve typical states of steady-state turbulence. It provides the simplest paradigm for fluctuations in the cold, collisional edge of toroidal devices and has been discussed by a number of workers [14, 21, 22].

Further nonlinear equations of interest to research on strongly magnetized plasmas are discussed in the review article by Krommes [14].

\section{PLASMA APPLICATIONS OF STATISTICAL METHODS}

Much evidence from both analysis and simulation shows that steady-state gyrokinetic microturbulence can exist, in agreement with experimental observations. Statistical descriptions are thus appropriate. Before turning in Sec. 4 to the main topic, the statistical description of convective cells, I wish to briefly mention some of the other applications for which statistical formalism has been used in plasma physics. For a much more complete review, see Ref. 14 .

\subsection{Gyrokinetic noise}

One method of solution of the gyrokinetic equation is by the so-called particle-in-cell technique (reviewed for unmagnetized plasmas in Ref. 23). A large number of particles is distributed at $t=0$ (either randomly or by a quiet start ${ }^{5}$ ). They are integrated along the characteristic trajectories of the gyrokinetic equation for a small time step, then the charge density is calculated by distributing the particles onto a spatial grid and the potential is updated by solving the gyrokinetic Poisson equation; the process is then repeated. ${ }^{6}$ The procedure is a Monte Carlo sampling method [25]; as such, one must contend with sampling

\footnotetext{
5 The general problem of initializing a particle distribution subject to various constraints is interesting and nontrivial. A Monte Carlo scheme particularly appropriate for generating states of negative temperature was discussed and analyzed in Ref. 24.

${ }^{6}$ In practice, one frequently uses a modification of this basic scheme wherein one solves only for perturbations away from a known, e.g., Maxwellian base state. Some remarks and historical references to this so-called $\delta f$ method can be found in Ref. 25 .
} 
noise. In unmagnetized plasmas, long-wavelength Fourier amplitudes are excited almost to thermal level. This would not be a problem if a physically realistic number of particles was used, since the microturbulent fluctuations are far above the thermal level; however, it is an important constraint for simulations, which can use only a restricted number of simulation particles. However, the fluctuation characteristics of gyrokinetic plasmas differ from those of the full many-body problem because high-frequency fluctuations have effectively been excised. The gyrokinetic fluctuation-dissipation theorem was studied in several papers by Krommes [15, 16, 26, 27]. As one would expect, the large dielectric constant of the gyrokinetic vacuum leads to strongly suppressed gyrokinetic fluctuations. Gyrokinetic particle simulation in fully 3D and global toroidal geometry is now a major industry.

\subsection{Realizable statistical closures}

In view of the physical complexity of nonlinear plasma dynamics, it is not surprising that the theory and applications of statistical closures for plasmas have lagged far behind their Navier-Stokes counterparts. An attempt at a historical survey of the plasma theories was made in Ref. 14; see in particular the bibliographical timelines presented in Figs. 34-36 of that work, which clearly show the inflow of information to plasma turbulence theory from the other physics specialities. A very brief historical overview follows. For reference, the direct-interaction approximation (DIA) was proposed by Kraichnan in 1959 [28].

The quasilinear description of plasmas was discussed by Vedenov et al. [29] and Drummond and Pines [30] in the early 1960's. Various calculations on perturbative weakturbulence theory [31, 32] soon followed. The first attempts at renormalization were by Dupree [33, 34]. In 1967 Orszag and Kraichnan [35] critically analysed Dupree's 1966

resonance-broadening theory (RBT) and gave a thorough discussion of the Vlasov DIA; unfortunately, that important work was ignored for about a decade.

A resurgence of interest in renormalized formalisms for plasmas was stimulated by the seminal 1973 paper of Martin, Siggia, and Rose (MSR) [36]. Krommes [37] discussed how RBT and related approximate plasma theories were embedded in the more general formalism. Dubois and Espedal [38] provided important insights about the general form of the nonlinear plasma dielectric function. Many additional references and historical remarks can be found in Ref. 14. 
By considering a three-wave example, Krommes [39] noted early on that the DIA could provide an adequate description of the saturation level of drift waves coupled with a Hasegawa-Mima-like nonlinearity. This was no surprise to the neutral-fluid community in view of the seminal work of Kraichnan [40], but it was the first quantitative calculation of the full DIA in a plasma context.

For practical calculations on many-mode problems such as HM or HW, it was readily apparent that Markovian closures were preferred. The eddy-damped quasinormal Markovian (EDQNM) closure was known to be realizable for Navier-Stokes dynamics [41]. It was therefore an unwelcome surprise when numerical integrations of the EDQNM for certain DW problems demonstrated unrealizable behavior. This problem was studied at length by Bowman [42] in collaboration with M. Ottaviani and the present author. The problem was traced to the triad interaction coefficient $\theta_{\boldsymbol{k p q}}$, which becomes complex in the presence of linear waves; the triad interaction time $\operatorname{Re} \theta_{\boldsymbol{k p q}} \equiv \theta_{\boldsymbol{k p q}}^{r}$ can then easily become negative. This difficulty arises in the evolution of the EDQNM from an initial state. Bowman and collaborators argued pragmatically that one should abandon a faithful description of the transient evolution (in any event, nonrealizable behavior cannot be faithful) while constraining the initial-value problem to evolve to the steady state described by the EDQNM. While there are various ways of doing this for the statistical description of scalar fields, the challenge of implementing this constraint for the evolution of systems of coupled fields proved to be formidable. Bowman et al. [43] discussed a method that appears to work satisfactorily in practice; they called the algorithm the realizable Markovian closure (RMC). $\mathrm{Hu}$ and Krommes $[21,44]$ used the RMC to discuss the statistical dynamics of the HW system; excellent agreement was found between direct numerical simulations and the closure predictions of the particle flux and wave-number spectra. Bowman [45] subsequently made related studies of HM dynamics; he also discussed and studied a realizable test-field model motivated by Kraichnan's work in Ref. 46.

The numerical closure studies that directly stemmed from Bowman's pioneering work are the most complete and recent in the context of plasma DW models. The successful comparisons between DNS and simple nonlinear DW paradigms such as HM and HW show that the essentials of second-order closures are reasonably well understood in plasma-physics contexts. At the present time, it appears to be hopeless to perform detailed closure studies on more realistic models involving nontrivial (e.g., toroidal) geometry, three dimensions, 
and the many practical effects that are inevitably present in real devices; such problems are better addressed by large-scale numerical gyrokinetic simulations, which are ongoing.

One fertile area for future research involves the analytical study of intermittent statistics, which are frequently observed in the edges of toroidal devices. This difficult area might be addressed through the mapping-closure techniques originally suggested in Ref. 47 and developed by later authors (for references, see Ref. 14 and the recent paper by Takaoka [48]). However, application of mapping closure to HW-like systems involves major technical complications, including generalization to coupled fields and the difficulty of adequately handling the nonlocal relation between potential $\varphi$ and vorticity $\nabla_{\perp}^{2} \varphi$. Research in this area [49] is still at a very early stage, so will not be discussed further in the present paper.

\section{STATISTICAL DESCRIPTION OF LONG-WAVELENGTH FLOWS}

I now turn to recent research on the statistical description of the turbulent self-generation of long-wavelength flows. This subject is intimately related to the seminal calculations of eddy viscosity by Kraichnan [50]; I will describe some new insights that have emerged recently. The problem is also of considerable practical importance in fusion physics, since it impacts on the physics of saturation of DW turbulence and thus has implications for the magnitudes of steady-state transport.

In the fusion context, it is usual to distinguish various special cases of convective cells $\left(k_{\|}=0\right)$ : zonal flows (ZF's; $y$-directed $\boldsymbol{E} \times \boldsymbol{B}$ velocities arising from potentials with $k_{y}=0$ ); and streamers $\left(x\right.$-directed velocities arising from potentials with $\left.k_{x}=0\right)$. Streamers can cause direct transport of particles and heat across magnetic flux surfaces, but can be broken up by secondary instabilities [51]. Zonal flows do not cause such transport, but they can interact with the DW's and thereby indirectly regulate the level of turbulent transport. Diamond et al. [52] attempted an analytical description of zonal-flow generation. Their basic idea, which was well motivated, was to assume scale separation between short-wavelength DW's and long-wavelength ZF's and to use a weakly inhomogeneous wave kinetic equation (WKE) for the DW's $\left(k_{\|} \neq 0\right)$ to calculate energy transfer to the ZF's due to the weak modulation of the DW's by the ZF's. A central question is what quantity to use as the "plasmon density" in the WKE. Some insight was provided by Smolyakov and Diamond [53], who derived "action invariants" for various simple paradigms such as the generalized HME. 
However, that work did not address the question of how to proceed for systems of coupled PDE's such as the HW system. I will discuss an appropriate methodology in Sec. 4.3.

Motivated by Refs. 52 and 53, Krommes and Kim (KK) [54] performed a detailed study of the problem of convective-cell generation by generalized HM dynamics in the limit of disparate scales. That work proceeded directly from Markovian statistical closure, thus was closely related to the calculations of Kraichnan [50] on the asymptotic long-wavelength limit of eddy viscosity in two dimensions. As I will describe, the work of KK sheds further light on the calculations of both Kraichnan as well as Diamond et al. and Smolyakov and Diamond, and unifies all such calculations for scalar fields into a common framework. Subsequent work ${ }^{7}$ on coupled-field systems $[55,56]$ provided an elegant formalism that subsumes the scalar calculations and essentially completes the formal statistical description of long-wavelength CC generation by short-wavelength DW's. That is, however, only one part of the full problem of interacting DW's and CC's. I will not discuss the effect of the CC's on the DW's in this paper except to say that preliminary work on systematic bifurcation analysis of the transition to drift-wave turbulence has been accomplished [57]; that work involves the CC backreaction in a fundamental way.

\subsection{Asymptotic long-wavelength expansion of the EDQNM formula for coherent damping}

For the CC generation problem, I first define exactly what statistical quantity is to be computed. Consider a scalar amplitude equation

$$
\partial_{t} \psi_{\boldsymbol{k}}-L_{\boldsymbol{k}} \psi_{\boldsymbol{k}}=\frac{1}{2} \sum_{\Delta} M_{\boldsymbol{k p q}} \psi_{\boldsymbol{p}}^{*} \psi_{\boldsymbol{q}}^{*}
$$

In a Markovian description of homogeneous statistics, the spectral-balance equation for wave-number intensity $C_{\boldsymbol{k}}(t) \doteq\left\langle\left|\delta \psi_{\boldsymbol{k}}(t)\right|^{2}\right\rangle$ is

$$
\partial_{t} C_{\boldsymbol{k}}-2\left(\operatorname{Re} L_{\boldsymbol{k}}\right) C_{\boldsymbol{k}}+2\left(\operatorname{Re} \eta_{\boldsymbol{k}}\right) C_{\boldsymbol{k}}=2 F_{\boldsymbol{k}}
$$

\footnotetext{
7 At the May, 2003 conference that these Proceedings document, my talk focused on the work of Krommes and Kim [54]. Further progress on coupled systems was made during the subsequent half-year that elapsed before publication of these Proceedings was formalized. In the interest of utility and completeness, I will incorporate those latter results into the present discussion.
} 
where $\eta_{\boldsymbol{k}}$ describes coherent nonlinear response and $F_{\boldsymbol{k}}$ is the variance of the nonlinear noise $\widetilde{f}_{\boldsymbol{k}}$ in the Langevin amplitude equation

$$
\partial_{t} \psi_{\boldsymbol{k}}-L_{\boldsymbol{k}} \psi_{\boldsymbol{k}}+\eta_{\boldsymbol{k}} \psi_{\boldsymbol{k}}=\widetilde{f}_{\boldsymbol{k}}(t)
$$

When I specialize to the DW-CC problem, I shall use $\boldsymbol{k}$ for the DW's and $\boldsymbol{q}$ for the CC's; I always work in the limit $\epsilon \doteq q / k \ll 1$. Then the CC growth rate $\gamma_{\boldsymbol{q}}$ is defined by

$$
\gamma_{\boldsymbol{q}} \equiv \gamma_{\boldsymbol{q}}^{\mathrm{nl}} \doteq-\operatorname{Re} \eta_{\boldsymbol{q}}
$$

The linear growth rate is, of course, $\gamma_{\boldsymbol{q}}^{\text {lin }}=\operatorname{Re} L_{\boldsymbol{q}}$. Thus the spectral balance equation for the CC's is

$$
\partial_{t} C_{\boldsymbol{q}}=2\left(\gamma_{\boldsymbol{q}}^{\operatorname{lin}}+\gamma_{\boldsymbol{q}}^{\text {nlin }}\right) C_{\boldsymbol{q}}+2 F_{\boldsymbol{q}}
$$

$F_{\boldsymbol{q}}$ is intrinsically positive, so one or both of $\gamma_{\boldsymbol{q}}^{\text {lin }}$ or $\gamma_{\boldsymbol{q}}^{\text {nlin }}$ must be negative in order that a steady state can be achieved. (Typically $\gamma_{\boldsymbol{q}}^{\text {lin }}$ is very small [58].) What I shall calculate in this work is the portion of $\gamma_{\boldsymbol{q}}^{\text {nlin }}$ due to interactions with short-wavelength $D W^{\prime}$ s. Except for a sign, this is exactly the statistical eddy viscosity in the asymptotic limit $\epsilon \ll 1$. If $\gamma_{\boldsymbol{q}}^{\mathrm{DW}}$ is positive, one is discussing negative eddy viscosity. This does not mean that the total $\gamma_{\boldsymbol{q}}^{\text {nlin }}$

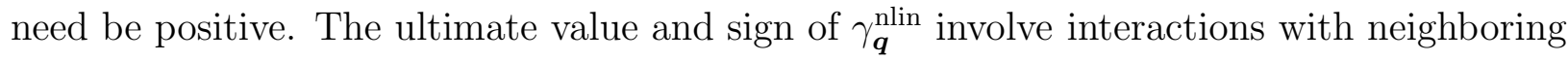
scales as well as distant ones, and the details cannot be available until all of the fully selfconsistent modal interactions are analyzed. Such calculations have not been published as yet.

According to the EDQNM, one has

$$
\gamma_{\boldsymbol{q}}=-\sum_{\Delta} M_{\boldsymbol{q} \boldsymbol{k} \boldsymbol{p}} M_{\boldsymbol{k} \boldsymbol{p} \boldsymbol{q}} \theta_{\boldsymbol{q} \boldsymbol{k} \boldsymbol{p}}^{r} C_{\boldsymbol{p}}
$$

Here $\sum_{\Delta}$ denotes the sum over all triangles such that $\boldsymbol{k}+\boldsymbol{p}+\boldsymbol{q}=\mathbf{0}$, and $\theta_{\boldsymbol{q} \boldsymbol{k} \boldsymbol{p}}$ is the triad interaction coefficient whose real part $\theta^{r}$ defines the triad interaction time. In the usual EDQNM, $\theta$ is a symmetric function of its arguments. ${ }^{8}$ The goal is to expand $\gamma_{\boldsymbol{q}}$ for $q \ll k, p$. In detail, this is somewhat tedious because if $\boldsymbol{q}$ is fixed, then $\boldsymbol{p}$ varies with $\boldsymbol{k}$. The integration domain is thus complicated (Fig. 4) and one must be very careful. The details were presented in Ref. 54, first for isotropic statistics (a test case that is closely related to

\footnotetext{
${ }^{8}$ Bowman [43] used an asymmetric function in his RMC.
} 
the calculations of Ref. 50), then for the more general and realistic anisotropic case. For the case of the GHME, the final result is

$$
\begin{gathered}
\gamma_{\boldsymbol{q}}=-\underbrace{\frac{2}{\alpha+q^{2}}}_{(\mathrm{a})} \sum_{\boldsymbol{k}} \underbrace{|\widehat{\boldsymbol{z}} \cdot(\boldsymbol{q} \times \boldsymbol{k})|^{2}}_{(\mathrm{b})} \underbrace{\theta_{\boldsymbol{k},-\boldsymbol{k}, \boldsymbol{q}}^{r}}_{(\mathrm{c})} \\
\times \underbrace{\frac{\boldsymbol{q} \cdot \boldsymbol{k}}{\left(1+k^{2}\right)^{2}}}_{(\mathrm{d})} \underbrace{\boldsymbol{q} \cdot \frac{\partial \mathcal{Z}_{\boldsymbol{k}}}{\partial \boldsymbol{k}}}_{(\mathrm{e})} .
\end{gathered}
$$

Here $\mathcal{Z}_{\boldsymbol{k}} \doteq\left(1+k^{2}\right)^{2}\left\langle\left|\delta \varphi_{\boldsymbol{k}}\right|^{2}\right\rangle$. In the next section I will describe a more physical algorithm that recovers this result. However, it is useful now to list the physical interpretation of each term:

(a) possibly nonadiabatic CC response;

(b) nonlinear advection of the DW's by the CC's;

(c) interaction time between the DW's and the CC's;

(d) DW wavevector refraction due to CC modulation;

(e) conservation of $\mathcal{Z}$ by the DW's due to CC modulation.

At this point, the interpretation of at least term $(d)$ is probably unclear. This will be explained by the physical algorithm to be discussed next.

\subsection{Weakly inhomogeneous spectral kinetics and the convective-cell growth rate}

Given the rigorous and systematically derived result (24), one can discuss various physical algorithms that reproduce it [54]. The basic idea, first proposed in Ref. 52, is to use a weakly inhomogeneous spectral balance equation or "wave kinetic equation" to describe the modification of the short-wavelength DW statistics due to the long-wavelength CC modulation. In Diamond's formalism, it was important that the proper quantity $\mathcal{Z}$ was used as the plasmon density evolved by the WKE, and Smolyakov and Diamond [53] discussed the appropriate form of $\mathcal{Z}$ for several popular scalar PDE's. Working with a particular $\mathcal{Z}$ in the WKE amounts to a particular ( $\boldsymbol{k}$-weighted) choice of dependent field variable in which to write the spectral balance equation. However, general statistical theory does not constrain the choice of dependent variable; moreover, for systems of coupled fields with multiple conserved quantities it might appear that the choice of dependent variables would 
be overconstrained. Such paradoxes led KK to reexamine the foundations of the spectral balance equation in the presence of weak inhomogeneity.

\subsubsection{General remarks about weakly inhomogeneous statistics}

For classical physics, the general problem of weak inhomogeneity (and weak nonstationarity) was addressed in the important paper of Carnevale and Martin [59] by means of multiple-scale expansions in both space and time. Focus on the spatial problem for simplicity. Then the general two-point correlation function $C\left(\boldsymbol{x}, \boldsymbol{x}^{\prime}\right)$ can be rewritten in terms of the sum and difference variables $\boldsymbol{X} \doteq \frac{1}{2}\left(\boldsymbol{x}+\boldsymbol{x}^{\prime}\right)$ and $\boldsymbol{\rho} \doteq \boldsymbol{x}-\boldsymbol{x}^{\prime}$ :

$C\left(\boldsymbol{x}, \boldsymbol{x}^{\prime}\right) \equiv C(\boldsymbol{\rho} \mid \boldsymbol{X})$. It is assumed that $C$ varies rapidly with $\boldsymbol{\rho}$ but slowly with $\boldsymbol{X}$. Let $\widehat{C}$ represent the abstract operator whose $\boldsymbol{x}$-space matrix element is $C\left(\boldsymbol{x}, \boldsymbol{x}^{\prime}\right)$. In general, linear operators act nonlocally on $C$, e.g., $(\widehat{L} \widehat{C})\left(\boldsymbol{x}, \boldsymbol{x}^{\prime}\right) \equiv \int d \overline{\boldsymbol{x}} L(\boldsymbol{x}, \overline{\boldsymbol{x}}) C\left(\overline{\boldsymbol{x}}, \boldsymbol{x}^{\prime}\right)$. According to CM, when this expression is expanded through first order in a weak inhomogeneity (and Fourier transformed with respect to $\boldsymbol{\rho})$, the result is

$$
\widehat{L} \widehat{C} \rightarrow L_{\boldsymbol{k}}(\boldsymbol{X}) C_{\boldsymbol{k}}(\boldsymbol{X})+i\left\{L_{\boldsymbol{k}}, C_{\boldsymbol{k}}\right\}
$$

where the Poisson bracket for weak inhomogeneity is

$$
\begin{aligned}
\{A, B\} & \doteq \frac{\partial A}{\partial \boldsymbol{X}} \cdot \frac{\partial B}{\partial \boldsymbol{k}}-\frac{\partial A}{\partial \boldsymbol{k}} \cdot \frac{\partial B}{\partial \boldsymbol{X}} \\
& =\frac{\partial}{\partial \boldsymbol{X}} \cdot\left(A \frac{\partial B}{\partial \boldsymbol{k}}\right)-\frac{\partial}{\partial \boldsymbol{k}} \cdot\left(A \frac{\partial B}{\partial \boldsymbol{X}}\right)
\end{aligned}
$$

[I will object to the result (25) momentarily.] The form (26b) shows that this bracket is conservative in the sense that it is annihilated by integration over $\boldsymbol{X}$ and summation over $\boldsymbol{k}$ (this pair of operations is denoted by an overline). That is,

$$
\overline{\{A, B\}}=0 \text {. }
$$

Thus under the action of a linear operator, which I now write as $L=-i \Omega$, the contribution of weak inhomogeneity to the evolution of the spectral density is apparently

$$
\partial_{T} C_{\boldsymbol{k}}=\left\{\Omega_{\boldsymbol{k}}, C_{\boldsymbol{k}}\right\}
$$

I have introduced $\Omega$ because in the application to the $\mathrm{CC}$ generation problem the relevant operator stems from the nonlinear advection and is generally purely real in $\boldsymbol{k}$ space. For 
example, if one uses the generalized HME to consider DW advection by a long-wavelength CC (denoted by an underline) with velocity $\underline{\boldsymbol{V}}_{E}(\boldsymbol{X})$, the relevant operator is

$$
\widehat{\Omega}=-i\left(1-\nabla_{\perp}^{2}\right)^{-1} \underline{\boldsymbol{V}}_{E} \cdot \nabla\left(1-\nabla_{\perp}^{2}\right),
$$

whose Fourier transform for constant $\boldsymbol{V}_{E}$ is

$$
\Omega_{\boldsymbol{k}}=\left(1+k_{\perp}^{2}\right)^{-1} \boldsymbol{k} \cdot \underline{\boldsymbol{V}}_{E}\left(1+k_{\perp}^{2}\right)=\boldsymbol{k} \cdot \underline{\boldsymbol{V}}_{E} .
$$

For the interaction between DW's and CC's, Smolyakov and Diamond [53] showed that a certain quantity $\mathcal{Z}$ was conserved. (In Sec. $4.3 \mathrm{I}$ will show that $\mathcal{Z}$ is a Casimir invariant in a noncanonical functional Hamiltonian description of the nonlinear physics.) However, for arbitrary choice of dependent variable, this contradicts the evolution equation (28) because barring that equation leads to $\partial_{T} \bar{C}=0$. That is, $C$ is apparently conserved regardless of the choice of variable. This conclusion is obviously incorrect.

The resolution of this paradox was given by KK, who took issue with the result (25) and its implication (28). Consider the situation where $\widehat{L}$ is the composition of two operators $\widehat{A}$ and $\widehat{B} ; \widehat{L}=\widehat{A} \widehat{B}$. Symmetry (or detailed calculation) dictates that the expression for $\widehat{L} \widehat{C}=\widehat{A} \widehat{B} \widehat{C}$ must contain the Poisson bracket of all of the operators taken in pairs:

$$
\begin{aligned}
\widehat{A} \widehat{B} \widehat{C} \rightarrow A_{\boldsymbol{k}} B_{\boldsymbol{k}} C_{\boldsymbol{k}}+i\left[\left\{A_{\boldsymbol{k}}, B_{\boldsymbol{k}}\right\} C_{\boldsymbol{k}}\right. \\
\left.\quad+A_{\boldsymbol{k}}\left\{B_{\boldsymbol{k}}, C_{\boldsymbol{k}}\right\}+\left\{A_{\boldsymbol{k}}, C_{\boldsymbol{k}}\right\} B_{\boldsymbol{k}}\right] .
\end{aligned}
$$

Upon combining the last two terms, this can be written as

$$
\left.\widehat{L} \widehat{C} \rightarrow L_{\boldsymbol{k}} C_{\boldsymbol{k}}+i \underline{\left[\left\{A_{\boldsymbol{k}}, B_{\boldsymbol{k}}\right\} C_{\boldsymbol{k}}\right.}+\left\{L_{\boldsymbol{k}}, C_{\boldsymbol{k}}\right\}\right]
$$

the underlined, nonconservative term (of first order in the weak gradient) was overlooked by CM. Thus, to the extent that $\widehat{L}$ is composed of noncommuting operators, $C$ is no longer conserved. Some other quantity, however, may be. Suppose that the quantity $\mathcal{Z}=\overline{\sigma_{\boldsymbol{k}}^{(\mathcal{Z})} C_{\boldsymbol{k}}}$ is known to be conserved, where $\sigma_{\boldsymbol{k}}^{(\mathcal{Z})}$ is a certain $\boldsymbol{k}$-dependent weight factor. It is then easy to see that the formalism is consistent provided that $\sigma^{(\mathcal{Z})}\{A, B\}-\left\{L, \sigma^{(\mathcal{Z})}\right\}=0$. [This must be appropriately generalized if $\widehat{L}$ is the composition of three or more operators. A good example is generalized HM dynamics, which involves the triple operator product given by Eq. (29).] That is, it must be the case that weak inhomogeneity leads to the spectral evolution equation

$$
\partial_{T} C_{k}=\left[\sigma_{\boldsymbol{k}}^{(\mathcal{Z})}\right]^{-1}\left\{\Omega_{\boldsymbol{k}}, \mathcal{Z}_{\boldsymbol{k}}\right\}=\left[\sigma_{\boldsymbol{k}}^{(\mathcal{Z})}\right]^{-1}\left\{\Omega_{\boldsymbol{k}}, \sigma_{\boldsymbol{k}}^{(\mathcal{Z})} C_{\boldsymbol{k}}\right\}
$$


where only the term explicitly related to the inhomogeneity is displayed. In the last form of Eq. (33), $\sigma_{\boldsymbol{k}}^{(\mathcal{Z})}$ may not be passed through the bracket because it is $\boldsymbol{k}$-dependent. The result (33) can be verified explicitly for specific PDE's such as the generalized HME [54].

\subsubsection{Modulated Reynolds stress, energy principles, and use of the Martin-Siggia-Rose formalism}

We wish to calculate $\gamma_{\boldsymbol{q}}$ by somehow invoking the theory of weakly inhomogeneous statistics. However, there is a technical problem: $\gamma_{\boldsymbol{q}}$ is nonzero even when the turbulence is entirely homogeneous. On the one hand, this is clear since, as I have stressed, $\gamma_{\boldsymbol{q}}$ represents only one part of the spectral balance equation. On the other hand, there certainly is a CC-DW interaction, and the intuition that a CC introduces a long-wavelength modulation seems compelling.

This paradox is reconciled in a well-known way by paying close attention to the distinction between an ensemble of CC's (distributed homogeneously) and a single test CC that perturbs the background turbulence. There is a precise analogy to the test-particle techniques of classical kinetic theory. Perturbations of background states are best handled by responsefunction techniques that generalize thermal-equilibrium fluctuation-dissipation theory [60]. The most elegant formulation was given in Ref. 36; I comment on it briefly here in order to emphasize the variational foundations of $\gamma_{\boldsymbol{q}}$, which lead rather directly to definitions of $\gamma_{\boldsymbol{q}}$ in terms of perturbed Reynolds stress and second-order variations of a certain energy functional.

Although it is unnecessary, consider statistically homogeneous turbulence for definiteness. In this case, all mean fields can be taken to vanish. A standard way of breaking the symmetry is to add an arbitrary, statistically sharp external source function $\widehat{\eta}_{\boldsymbol{k}}(t)$ to the right-hand side of the dynamical equation (18). This inevitably induces nonvanishing mean fields $\langle\psi\rangle_{\widehat{\eta}}$ that depend functionally on $\widehat{\eta}$. Furthermore, other statistical observables such as the two-point correlation function $C$ and response function $R$ also depend functionally on $\widehat{\eta}$. One can use the mean field rather than $\widehat{\eta}$ as the control parameter, so one can discuss such functional dependences as $C[\langle\psi\rangle]$.

MSR showed that it is also useful to introduce a source $\eta$ that appears in an equation adjoint to that for $\psi$. Then $n$-point cumulants of $\psi$ generate $(n+1)$-point cumulants by functional differentiation: $C_{\eta}^{(n+1)}(1,2, \ldots, n, n+1)=\delta C_{\eta}^{(n)}(1,2, \ldots, n) / \delta \eta(n+1)$. They also 
emphasized that proceeding in this way merely leads to an unclosed cumulant hierarchy, the generalization of the BBGKY hierarchy of classical many-body kinetic theory. To effect closure, one must introduce response functions. For the details, see Ref. 14 and references therein. The result is that the two-point response function is

$$
R\left(1 ; 1^{\prime}\right)=\left.\frac{\delta\langle\psi\rangle_{\boldsymbol{\eta}}(1)}{\delta \widehat{\eta}\left(1^{\prime}\right)}\right|_{\boldsymbol{\eta}=\mathbf{0}},
$$

where $\boldsymbol{\eta} \doteq(\eta, \widehat{\eta})^{T}$. Furthermore, the triplet correlation that appears in the evolution equation for $R$ can be shown by means of the functional chain rule to be equal to $\Sigma R$, where

$$
\Sigma\left(1 ; 1^{\prime}\right)=\left.\frac{\delta\langle\langle N\rangle\rangle_{\boldsymbol{\eta}}(1)}{\delta\langle\psi\rangle_{\boldsymbol{\eta}}\left(1^{\prime}\right)}\right|_{\boldsymbol{\eta}=\mathbf{0}} .
$$

Here $\langle\langle N\rangle\rangle$ is the cumulant part of the nonlinear term in the original dynamical equation. This rewriting of the triplet correlation function effects the elimination of the disconnected graphs in the derivation of the famous Dyson equation [61]; the method of sources discussed here is due to Schwinger. $\gamma_{\boldsymbol{q}}$ is a Markovian version of $-\Sigma$.

Now $\langle\langle N\rangle\rangle$ is just the generalization of the (divergence of the) Reynolds stress of NavierStokes theory to arbitrary nonlinear dynamics. One thus understands that $\gamma_{\boldsymbol{q}}$ is not determined from the Reynolds stress itself, but rather from a perturbed stress modulated by the CC's. Failure to appreciate this point can lead to paradoxes and incorrect results. Consider, for example, the GHME problem. There the nonlinear effect on the CC's is the advection of vorticity (not of velocity, as in the usual Navier-Stokes equation). The appropriate nonlinear effect driving the CC's is therefore (with primes denoting the DW's)

$$
\begin{aligned}
\left\langle\boldsymbol{V}_{E}^{\prime} \cdot \nabla \nabla^{2} \varphi^{\prime}\right\rangle=\left(\partial_{x x}-\partial_{y y}\right)\left\langle v_{x}^{\prime} v_{y}^{\prime}\right\rangle & \\
& +\partial_{x y}\left(\left\langle v_{y}^{\prime 2}\right\rangle-\left\langle v_{x}^{\prime 2}\right\rangle\right) .
\end{aligned}
$$

Here the last result, involving the physical Cartesian components of the $\boldsymbol{E} \times \boldsymbol{B}$ velocity, follows by integration by parts assuming homogeneous statistics. Now consider isotropic DW statistics for definiteness. In this case one has $\left\langle v_{y}^{\prime 2}\right\rangle=\left\langle v_{x}^{\prime 2}\right\rangle$ and $\left\langle v_{x}^{\prime} v_{y}^{\prime}\right\rangle=0$. That is, the Reynolds stress vanishes for homogeneous, isotropic turbulence, which is well known. But the perturbed Reynolds stress on the DW's due to CC modulation does not vanish. The presence of a CC with wavevector $\boldsymbol{q}$ introduces an anisotropy dependent on the direction of $\boldsymbol{q}$. Now both $\Delta\left\langle v_{y}^{\prime 2}\right\rangle \neq \Delta\left\langle v_{x}^{\prime 2}\right\rangle$ and $\Delta\left\langle v_{x}^{\prime} v_{y}^{\prime}\right\rangle \neq 0$. When those modulations are calculated 
(according to the algorithm to be described shortly), one is led to the proper answer (24). ${ }^{9}$

The analysis can be continued to demonstrate that $\gamma_{\boldsymbol{q}}$ can be obtained from the second functional derivative of a certain energy rate-of-change functional. That is, one can derive a generalized Poynting theorem. For HM dynamics, that is

$$
\partial_{t} \overline{\mathcal{E}}=\langle\underline{\boldsymbol{E}}\rangle \cdot \boldsymbol{\Gamma}
$$

where $\overline{\mathcal{E}}$ is the DW energy averaged over the long wavelengths and $\Gamma$ is the flux of DW vorticity. Note the presence of the induced mean field. As in the discussion of the specific example (36), the right-hand side of expression (37) would vanish for homogeneous turbulence in the absence of the symmetry-breaking $\widehat{\eta}$. In its presence, $\dot{\overline{\mathcal{E}}}$ is at least of second order in $\widehat{\eta}$ or $\langle\varphi\rangle$, and one can show [54] that

$$
\gamma_{\boldsymbol{q}}=-\left.\left(\frac{1}{\alpha+q^{2}}\right) \frac{\delta^{2} \dot{\overline{\mathcal{E}}}}{\delta\left\langle\varphi_{\boldsymbol{q}}\right\rangle \delta\left\langle\varphi_{\boldsymbol{q}}\right\rangle^{*}}\right|_{\boldsymbol{\eta}=\mathbf{0}} .
$$

Note that the differentiation is with respect to the induced mean field. A more definitive derivation of Eq. (38) follows from the Hamiltonian formalism to be discussed in Sec. 4.3.

What remains is to calculate $\dot{\overline{\mathcal{E}}}$ from the weakly inhomogeneous WKE as discussed in Sec. 4.2. Although $\mathcal{Z}$ is conserved under the DW-CC interaction, $\mathcal{E}$ is not. Upon assuming that the DW energy is related to the spectral density according to $\mathcal{E}_{\boldsymbol{k}}=\sigma_{\boldsymbol{k}}^{(\mathcal{E})} C_{\boldsymbol{k}}$, and upon defining $\rho_{\boldsymbol{k}} \doteq \sigma_{\boldsymbol{k}}^{(\mathcal{Z})} / \sigma_{\boldsymbol{k}}^{(\mathcal{E})}$, one may use Eq. (33) to find

$$
\partial_{T} \mathcal{E}_{\boldsymbol{k}}=\rho_{\boldsymbol{k}}^{-1}\left\{\Omega_{\boldsymbol{k}}, \rho_{\boldsymbol{k}} \mathcal{E}_{\boldsymbol{k}}\right\}=\left\{\Omega_{\boldsymbol{k}}, \mathcal{E}_{\boldsymbol{k}}\right\}+\left\{\Omega_{\boldsymbol{k}}, \ln \rho_{\boldsymbol{k}}\right\} \mathcal{E}_{\boldsymbol{k}}
$$

The first term is in conservative form and vanishes under the barring operation, but the last term describes a mean energy transfer from the DW's to the CC's. (The actual sign is not clear at this point.) The last Poisson bracket simplifies to $\nabla \Omega_{\boldsymbol{k}} \cdot \partial_{\boldsymbol{k}} \ln \rho_{\boldsymbol{k}}$ because $\rho_{\boldsymbol{k}}$ depends only on $\boldsymbol{k}$. Thus

$$
\begin{aligned}
\partial_{T} \overline{\mathcal{E}} & =\sum_{\boldsymbol{k}} \overline{\left[\nabla \Omega_{\boldsymbol{k}}(\boldsymbol{X}) \cdot \partial_{\boldsymbol{k}} \rho_{\boldsymbol{k}}\right] \mathcal{Z}_{\boldsymbol{k}}(\boldsymbol{X})} \\
& =\sum_{\boldsymbol{k}} \sum_{\boldsymbol{q}}\left(-i \boldsymbol{q} \cdot \partial_{\boldsymbol{k}} \rho_{\boldsymbol{k}}\right) \Omega_{\boldsymbol{k} ; \boldsymbol{q}}^{*} \mathcal{Z}_{\boldsymbol{k} ; \boldsymbol{q}}
\end{aligned}
$$

\footnotetext{
${ }^{9}$ Kim and Diamond [62] suggested that one should ignore the term in $\left\langle v_{y}^{2}\right\rangle-\left\langle v_{x}^{2}\right\rangle$ (on the grounds that it vanishes for isotropic statistics) while retaining the term in $\left\langle v_{x} v_{y}\right\rangle$. As discussed by Krommes [63], this argument is incorrect; if it is pursued [62], it leads to a formula for $\gamma_{\boldsymbol{q}}$ that is not properly invariant under rotations of $\boldsymbol{q}$.
} 
where Parseval's theorem was used in the last step in order to Fourier-decompose the $\boldsymbol{X}$ dependence of the CC's into the $\boldsymbol{q}$ variable. The functional derivatives required in Eq. (38) can now be readily performed; only the product $\Omega_{\boldsymbol{k} ; \boldsymbol{q}}^{(1)} \mathcal{Z}_{\boldsymbol{k} ; \boldsymbol{q}}^{(1)}$ contributes. For $\mathcal{Z}_{\boldsymbol{k}}^{(1)}$, one may use a variant of Eq. (33). That equation is not complete, however, since it seems to suggest that the individual spectral components $\partial_{T} \mathcal{Z}_{\boldsymbol{k}}$ would never reach a steady state. Such a state is achieved only by the interaction between the CC's and the DW's, and in Markovian closure theory the duration of that interaction is given by the triad interaction time $\theta_{\boldsymbol{q}, \boldsymbol{k},-\boldsymbol{k}}$. Therefore, guided by the rigorous asymptotic closure results, I heuristically generalize the first-order version of Eq. (33) to

$$
\left[\partial_{T}+\left(\theta_{\boldsymbol{q}, \boldsymbol{k},-\boldsymbol{k}}^{r}\right)^{-1}\right] \mathcal{Z}_{\boldsymbol{k} ; \boldsymbol{q}}^{(1)}=\left\{\Omega_{\boldsymbol{k} ; \boldsymbol{q}}^{(1)}, \mathcal{Z}_{\boldsymbol{k}}^{(0)}\right\}
$$

(The term $\left\{\Omega_{\boldsymbol{k}}^{(0)}, \mathcal{Z}_{\boldsymbol{k} ; \boldsymbol{q}}^{(1)}\right\}$ vanishes at $\langle\varphi\rangle=0$.) Now one can consider a true steady state in which $\partial_{T} \mathcal{Z}_{\boldsymbol{k}}=0$ and find that

$$
\mathcal{Z}_{\boldsymbol{k} ; \boldsymbol{q}}^{(1)}=\theta_{\boldsymbol{q}, \boldsymbol{k},-\boldsymbol{k}}^{r}\left\{\Omega_{\boldsymbol{k} ; \boldsymbol{q}}^{(1)}, \mathcal{Z}_{\boldsymbol{k}}^{(0)}\right\}
$$

Substitution of this result into Eq. (40b) leads to Eq. (24). The physical effect involved is identified by noting that

$$
\left\{\Omega^{(1)}, \mathcal{Z}^{(0)}\right\}=\nabla \Omega^{(1)} \cdot \partial_{\boldsymbol{k}} \mathcal{Z}^{(0)}
$$

In the full equation for $\partial_{T} \mathcal{Z}$, the associated characteristic equation is

$$
d \boldsymbol{k} / d t=-\nabla \Omega
$$

which describes a refractive change in wavevector due to the weak inhomogeneity induced by the modulation. This effect gives rise to term (d) in Eq. (24).

This calculation of $\gamma_{\boldsymbol{q}}$ and the result (24) are very closely related to Kraichnan's classic calculation of 2D eddy viscosity [50]. He used the NSE, whereas Eq. (24) pertains to the HME. However, the plasma problem may be readily reduced to the Navier-Stokes one by removing the term describing adiabatic electron response [the 1 in the factor $\left(1+k^{2}\right)$ ]. Upon doing that, KK showed that Eq. (24) reduces precisely to the result of Kraichnan (who considered only homogeneous, isotropic statistics). It is then instructive to compare Kraichnan's physical explanation with the above discussion based on the WKE. Kraichnan considered a special case; however, it can be seen from study of Ref. 50 that Kraichnan understood clearly that the underlying mechanism was wavevector refraction. The merits 
of the present analysis employing the WKE are that the analyis is quite general, holds for arbitrary anisotropic statistics, and has an elegant generalization to the case of multiple coupled fields, as discussed below in Sec. 4.3.

For isotropic statistics, the eddy viscosity calculated by Kraichnan and also by KK for the HME is negative $\left(\gamma_{\boldsymbol{q}}>0\right)$. In the Appendix of Ref. 54, KK commented on some literature in which a positive eddy viscosity was apparently found. The reconciliation was that the present calculations pertain to a self-consistent turbulent steady state in which the total energy of the DW's plus CC's is conserved. The predictions of positive eddy viscosity arise from initial-value calculations in which the DW energy is held fixed; KK identified the specific term in the asymptotic analysis that is omitted in the initial-value calculations. For most purposes, it is the self-consistent, steady-state version of the formalism that is appropriate.

\subsection{Hamiltonian formalism}

The discussion to this point covers the material presented in the May, 2003 Conference represented by these Proceedings. Subsequently, new insights into, and generalizations of the formalism have been obtained. Although these have been detailed in other publications $[55,56]$, it is useful for completeness to briefly discuss them here as well.

An outstanding question is, How does one generalize the heuristic algorithm discussed in Sec. 4.2.2 to the important physical situation of multiple coupled fields, e.g., the HW equations? Systems of PDE's usually possess multiple invariants. Which one, if any, plays the preferred role of the $\mathcal{Z}$ in the scalar case? The answer is not immediately clear.

In order to address these questions, it is important to realize that the $\mathrm{CC}$ generation process is entirely nonlinear. The details of linear physics enter the expression for the CC growth rate only indirectly through the properties of the triad interaction time $\theta^{r}$. Although the linear evolution matrix may be essentially arbitrary (in particular, its eigenvalues may be complex, representing linear forcing and dissipation), the nonlinear terms are usually conservative. That is certainly true if the nonlinear behavior arises from $\boldsymbol{E} \times \boldsymbol{B}$ advection. A powerful way of deriving conservative nonlinear Eulerian PDE's is to use a Hamiltonian formalism. This provides new insights even for a scalar PDE such as the Hasegawa-Mima equation. Moreover, a generalization to multiple coupled fields leads to a particularly efficient 
representation of the $\gamma_{\boldsymbol{q}}$ for that case.

\subsubsection{Hamiltonian description of Eulerian partial differential equations}

A Hamiltonian description of certain Eulerian PDE's was first given by Morrison and Greene [64]. An extensive amount of subsequent additional work was reviewed by Morrison in Ref. 65. First consider the scalar case for simplicity. Let $\psi(\boldsymbol{x}, t)$ be the dependent variable. The basic idea is to exhibit a Hamiltonian functional $\mathcal{H}[\psi]$ and a Poisson-bracket functional $\{\cdot, \cdot\}$ such that the conservative PDE can be represented in the form

$$
\partial_{t} \psi=\{\psi, \mathcal{H}\}
$$

This generalizes to an uncountably infinite number of spatial points $\boldsymbol{x}$ the familiar equation of finite-dimensional, noncanonical Hamiltonian dynamics $\dot{z}^{i}=\left\{z^{i}, H\right\}$ (on which, for example, the modern gyrokinetic formalism [7] is based).

Consistency of the formalism requires that the bracket be antisymmetric $(\{A, B\}=$ $-\{B, A\})$ and obey the Jacobi identity $(\{\{A, B\}, C\}+\{\{B, C\}, A\}+\{\{C, A\}, B\}=0)$, where $A, B$, and $C$ are arbitrary functionals.

As an example, consider the 2D NSE for vorticity $\Omega=\nabla^{2} \varphi$ :

$$
\partial_{t} \Omega+\boldsymbol{V} \cdot \nabla \Omega=0
$$

where $\boldsymbol{V}=\widehat{\boldsymbol{z}} \times \boldsymbol{\nabla} \varphi$. It is well known that $\boldsymbol{V} \cdot \boldsymbol{\nabla} \Omega=[\varphi, \Omega]$, where

$$
[A, B] \doteq \widehat{\boldsymbol{z}} \cdot \nabla A \times \nabla B
$$

Now consider the Hamiltonian

$$
\mathcal{H}[\Omega]=\frac{1}{2} \overline{\Omega\left(-\nabla^{-2}\right) \Omega}
$$

(integration by parts shows that $\mathcal{H}=\frac{1}{2} \overline{|\boldsymbol{V}|^{2}}$ ) and the bracket

$$
\{A, B\}=\overline{\psi\left[\frac{\delta A}{\delta \psi}, \frac{\delta B}{\delta \psi}\right]} .
$$

One readily verifies that $\{\Omega, \mathcal{H}\}=-[\varphi, \Omega]$, so Eq. (45) reproduces Eq. (46).

In considering the generalization of this result to multiple-field systems, Krommes and Kolesnikov [55] restricted their attention to PDE's whose nonlinearities can be represented by the square Poisson bracket (47). That includes the $\boldsymbol{E} \times \boldsymbol{B}$ nonlinearity (as in, for example, 
the HWE's) as well as the field-line bending terms that arise in weakly electromagnetic generalizations of electrostatic models. Thus, they considered the Hamiltonian functional

$$
\mathcal{H}[\boldsymbol{\psi}]=\frac{1}{2} \overline{\psi^{i} g_{i j} \psi^{j}}
$$

and the Lie-Poisson bracket

$$
\{A, B\}=\overline{S^{i j}\left[\frac{\delta A}{\delta \psi^{i}}, \frac{\delta B}{\delta \psi^{j}}\right]} .
$$

Here $g_{i j}$ and $S^{i j}$ are symmetric matrices. $g$ is taken to be independent of $\boldsymbol{\psi}$, so $\mathcal{H}$ can be considered to be a generalized kinetic energy. $g$ plays the role of a metric tensor that can be used to raise and lower indices; for example, the covariant component of $\boldsymbol{\psi}$ is $\psi_{i}=g_{i j} \psi^{j}$. Thus one has the compact covariant expression

$$
\mathcal{H}[\boldsymbol{\psi}]=\frac{1}{2} \overline{\psi^{i} \psi_{i}}
$$

$\mathrm{S}$ is taken to be linear in $\boldsymbol{\psi}$, viz.

$$
S^{i j}[\boldsymbol{\psi}]=S^{i j} \psi^{k}
$$

$\mathrm{S}$ is called the structure matrix and the $S^{i j}{ }_{k}$ are called the structure constants. In order that the Jacobi identity be satisfied, the matrix $T^{i j k}{ }_{m} \doteq S^{i j}{ }_{l} S^{l k}{ }_{m}$ must be fully symmetric in $i, j$, and $k(\forall m)$.

It is readily seen that the nonlinear dynamics conserve the Hamiltonian $\mathcal{H}$. Other quantities may be conserved as well. In particular, a quantity that is conserved independently of the form of $\mathcal{H}$ is called a Casimir invariant $\mathcal{Z}$. Thus, the conservation of a Casimir depends only on the properties of the Poisson bracket; with the particular choice of bracket given above, Casimir conservation is encoded in the properties of the structure tensor $S^{i j}{ }_{k}$. Consider, for example, how that bracket conserves both $\mathcal{H}=\frac{1}{2} \overline{\psi^{i} \psi_{i}}$ and the prospective Casimir $\mathcal{Z}=\frac{1}{2} \overline{\psi^{i} \psi^{i}}$. In component form, the vector generalization of Eq. (45) is

$$
\partial_{t} \psi^{i}=S_{k}^{i j}\left[\psi^{k}, \psi_{j}\right]
$$

One has

$$
\partial_{t} \mathcal{H}=\overline{\dot{\psi}^{i} \psi_{i}}=S^{i j} \overline{\left[\psi^{k}, \psi_{j}\right] \psi_{i}}=S^{i j} \overline{\psi^{k}\left[\psi_{j}, \psi_{i}\right]}=0
$$

the last result following from the contraction of a symmetric form (in contravariant indices) and an antisymmetric form (in covariant indices). Similarly,

$$
\partial_{t} \mathcal{Z}=\overline{\dot{\psi}^{i} \psi^{i}}=S^{i j}{ }_{k} \overline{\left[\psi^{k}, \psi_{j}\right] \psi^{i}}=S^{i j} \overline{{ }_{k} \overline{\left.\psi^{k}, \psi^{i}\right] \psi_{j}}} .
$$


Now the antisymmetric form is in contravariant indices. This expression will therefore vanish if $S^{i j}{ }_{k}=S^{k j}{ }_{i}$. This symmetry holds sometimes but not always.

Thus the conservation of the particular form $\mathcal{Z}=\frac{1}{2} \overline{\psi^{i} \psi^{i}}$ depends on the up-down symmetry properties of the structure tensor. That this particular form is not always conserved is understandable since $\psi^{i} \psi^{i}$ is not a covariant expression. That is, its form will change under a linear transformation of the field variables. Under that same transformation the symmetries of the structure tensor will also change. Thus there may exist a bilinear form in the $\psi^{i}$ 's that is a Casimir. Generally there are multiple Casimirs $\mathcal{Z}^{(n)}$. Of course, each such Casimir constrains the dynamics to move in a subspace of the full energy surface; see Fig. 4. We will see that the Casimirs figure importantly in the CC generation problem.

\subsubsection{Hamiltonian description of $\gamma_{\boldsymbol{q}}$}

In order to use this formalism to calculate the generation rate of CC's, one needs to (i) obtain a generalized energy theorem; (ii) represent the multivariate spectral balance equation in Hamiltonian form; (iii) use that equation to calculate the requisite functional derivatives. To derive (i), one begins by projecting the dynamical equation (54) into the DW subspace (denoted by primes) and the CC subspace (denoted by an underline). (An underline can be interpreted as integration over $z$, i.e., extracting the $k_{\|}=0$ component.) For example, one has

$$
\partial_{t} \psi^{i \prime}=S_{k}^{i j}\left(\left[\psi^{k \prime}, \underline{\psi}_{j}\right]+\left[\underline{\psi}^{k}, \psi_{j}^{\prime}\right]\right)+\text { self-interaction terms. }
$$

Next, an equation for the quadratic DW and CC energies can be obtained by contracting Eq. (57) with $\psi_{i}$ and barring the resulting equation. The details are given in Ref. 55; the result is that the DW power loss is

$$
\partial_{t} \overline{\mathcal{E}}=G^{i} P_{i}
$$

where $\boldsymbol{G}$ is the cumulant part of the mean nonlinearity and $\underline{\boldsymbol{P}}$ is the mean induced field. This covariant result should be compared to formula (37).

One may now prove an important theorem about the spectral dynamics. In Eq. (57), the first term on the right-hand side describes advection of the DW's by the CC's; the second term describes the advection of the CC's by the DW's. This latter effect is small and will be neglected. Because the retained interaction involves the covariant component $\underline{\psi}_{j}$ (which is $\left.\delta \mathcal{H} / \delta \psi^{j}\right)$, the DW's conserve the same Casimirs as the full dynamics. This result is the 
generalization to multifield systems of the formulas given in Ref. 53 for conserved quantities in certain scalar models. That is, in the presence of CC modulation and to lowest order in the scale-separation parameter $\epsilon$, the DW's conserve the Casimir invariant(s).

In the presence of multiple Casimir invariants, there is no a priori invariant that is preferred (i.e., is the leading candidate for an action density in a scalar WKE). In lieu of other physical information, one should treat all Casimirs on equal footing. The way to accomplish that is simply to work with the tensor spectral-balance equation, i.e., the equation for the DW $\partial_{t} C_{\boldsymbol{k}}^{i j}$. The calculation is sketched in Ref. 55. The final result is $\left(\Sigma^{i j}\right)^{H}=H^{(i j)}\left(\Sigma^{i j}\right)$, where $H^{(i j)}$ denotes the Hermitian part with respect to the indices $i$ and $j$, and

$$
\begin{aligned}
\Sigma_{\boldsymbol{q}}^{i j}=-\sum_{\boldsymbol{k}} & d^{2} S^{i r}{ }_{k}\left[(\partial+2 i) g_{r s}\right] \\
& \times \widehat{\boldsymbol{\theta}}^{k s}{ }_{\bar{k} \bar{s}} H^{(\bar{k} \bar{s})}\left[S^{j \bar{k}}{ }_{l}(\partial+2 i) C_{\boldsymbol{k}}^{l \bar{s}}\right]
\end{aligned}
$$

$d \doteq \widehat{\boldsymbol{z}} \cdot \boldsymbol{q} \times \boldsymbol{k}$, and $\partial \doteq \boldsymbol{q} \cdot \partial_{\boldsymbol{k}}$. This result should be compared to the scalar result (24). The general similarities in form are obvious. The terms proportional to $2 i$ are associated with off-diagonal correlations and vanish in the scalar case. The effect of wave-number refraction show up here as the derivative of the metric tensor $g$. The scalar triad interaction time has been heuristically generalized to a fourth-rank tensor $\widehat{\boldsymbol{\theta}}$; further discussion is given in Sec. 4.3.3. Most strikingly, the new result involves $\partial C$, not the derivative of any particular Casimir. This may be troubling because it may not be apparent how the limit of a scalar system is achieved; this will also be discussed below. But such a form should really be expected because no Casimir was given preferred treatment; conservation of all Casimirs is automatically built into the result (59). This is true even though one may not explicitly know the forms of the $\mathcal{Z}^{(n)}$.

Several examples of physically relevant PDE's that can be treated by this Hamiltonian formalism were given in Ref. 55; they include a two-field model describing ion-temperaturegradient-driven fluctuations and a three-field model describing weakly electromagnetic corrections to the electrostatic HW system.

One way of using the formula (59) is to identify a small parameter $\beta$ and to calculate the lowest-order corrections to the zeroth-order result. For example, one could calculate $\gamma_{\boldsymbol{q}}$ for the two-field electrostatic HW model, then recalculate it for the weakly electromagnetic three-field model (using as small parameter the normalized plasma pressure $\beta$ ). Typically 
$\beta$ corrections show up in the metric tensor $g$, so the procedure is in principle straightforward for fixed $\widehat{\boldsymbol{\theta}}$ and $\mathrm{C}$. However, it is inconsistent to hold those quantities fixed because the backreaction of the CC's on the DW's causes $\beta$-related modifications to the DW spectrum. Those cannot be calculated within the present formalism; a fully self-consistent theory is required.

\subsubsection{The tensor triad interaction time}

Left undetermined in Eq. (59) was the form of the triad interaction tensor $\widehat{\boldsymbol{\theta}}$. That object is already nontrivial in the scalar case due to issues relating to random Galilean invariance [54]. Its tensor generalization to multiple fields may appear daunting. For some discussions of tensor interaction times, see Ref. 43.

One important cross check is to verify that formula (59) reduces properly to a singlefield limit. The electrostatic HW model permits such a limit, which may be obtained by

letting $\widehat{\delta}$ approach infinity. In that limit, consistent balance requires that the terms $\widehat{\delta}(\varphi-n)$ remain finite, i.e., that $n \rightarrow \varphi$ (adiabatic electron response). Subtraction of Eq. (17a) from Eq. (17b) then leads to the HM equation if the dissipative terms are ignored. But does Eq. (59) approach Eq. (24) in that same limit? An important feature of Eq. (24) is that it involves $\partial \mathcal{Z}_{\boldsymbol{k}}$. However, formula Eq. (59) involves $\partial \mathrm{C}$. It is easy to see that if $\widehat{\boldsymbol{\theta}}$ is taken to be diagonal (an assumption that has been frequently made for simplicity [66]), the appropriate limit cannot be obtained. Instead, it can be shown that as $\widehat{\delta} \rightarrow \infty \widehat{\boldsymbol{\theta}}$ approaches a singular matrix whose size is dictated by the scalar $\theta_{\boldsymbol{q}, \boldsymbol{k},-\boldsymbol{k}}$ and whose structure guarantees the adiabatic relationship between $n$ and $\varphi$; off-diagonal components are essential. When the details are carried through, it can ultimately be shown [67] that the proper HM limit is, in fact, achieved. This is a necessary cross check. Unfortunately, it suggests that one must be extremely careful with the use of the tensor $\widehat{\boldsymbol{\theta}}$; cavalier assumptions here may seriously vitiate the fidelity of the overall calculation. Further work remains to be done on this topic.

\section{DISCUSSION}

I have tried to provide an overview of the general state of affairs of some basic aspects of statistical plasma physics as motivated by confinement in strongly magnetized plasmas. 
I emphasized (i) the use of the gyrokinetic formalism; (ii) the development of realizable Markovian closures and their applications to simple nonlinear plasma paradigms; (iii) the statistical description of convective cells (including zonal flows). Although the latter topic only provides part of the story of self-consistent turbulence, it is quite rich in detail and unifies various technical tools, including asymptotic expansion of Markovian statistical closure, weakly inhomogeneous spectral evolution equations, the use of field-theoretic techniques, the derivation of energy variational principles, and the Hamiltonian formulation of nonlinear PDE's.

In general terms, what remains is a detailed investigation of the backreaction of the CC's on the DW's. An analytical formulation of that problem is nontrivial. Energetic consistency in a steady state demands that comparable-sized scales be included in the mode coupling; those are not amenable to asymptotic techniques. Drift-wave physics is intrinsically anisotropic, and any sort of analytically tractable model will significantly underrepresent the complexity of real devices. Numerical simulations will be of obvious help, and those are planned by a number of groups. The importance of the drift-wave problem, not only for the understand of confinement in fusion plasmas but also for its relevance to geophysics, means that the subject will remain of considerable interest for an extended period of time.

\section{Acknowledgments}

I am grateful for the hospitality of S. Shivamoggi and the University of Central Florida during the period of the Conference represented by these Proceedings. This work was supported by U. S. Dept. of Energy Contract No. DE-AC02-76-CHO-3073.

[1] Rosenbluth, M. N. (1965). Microinstabilities. In: Plasma Physics. International Atomic Energy Agency, pp. 485-513.

[2] Chandrasekhar, S. (1960). Plasma Physics. Chicago: U. of Chicago Press.

[3] Antonsen, T. M. Jr., Lane, B. (1980). Kinetic equations for low frequency instabilities in inhomogeneous plasmas. Phys. Fluids 23:1205-1214.

[4] Catto, P. J., Tang, W. M., Baldwin, D. E. (1981). Generalized gyrokinetics. Plasma Phys. 23:639-650. 
[5] Frieman, E. A., Chen, L. (1982). Nonlinear gyrokinetic equations for low-frequency electromagnetic waves in general plasma equilibria. Phys. Fluids 25:502-508.

[6] Lee, W. W. (1983). Gyrokinetic approach in particle simulation. Phys. Fluids 26:556-562.

[7] Dubin, D. H. E., Krommes, J. A., Oberman, C. R., Lee, W. W. (1983). Nonlinear gyrokinetic equations. Phys. Fluids 26:3524-3535.

[8] Littlejohn, R. G. (1979). A guiding center Hamiltonian: A new approach. J. Math. Phys. $20: 2445-2458$.

[9] Cary, J. R., Littlejohn, R. G. (1983). Noncanonical Hamiltonian mechanics and its application to magnetic field line flow. Ann. Phys. (N.Y.) 151:1-34.

[10] Hahm, T. S. (1988). Nonlinear gyrokinetic equations for tokamak microturbulence. Phys. Fluids 31:2670-2673.

[11] Brizard, A. (1990). Nonlinear Gyrokinetic Tokamak Physics. PhD thesis: Princeton University.

[12] Qin, H. (1998). Gyrokinetic Theory and Computational Methods for Electromagnetic Perturbations in Tokamaks. PhD thesis: Princeton University.

[13] Dubin, D. H. E., Krommes, J. A. (1982). Stochasticity, superadiabaticity, and the theory of adiabatic invariants and guiding center motion. In: Horton, W., Reichl, L. E., Szebehely, V. G., eds. Long Time Prediction in Dynamics. New York: Wiley, pp. 251-280.

[14] Krommes, J. A. (2002). Fundamental statistical theories of plasma turbulence in magnetic fields. Phys. Rep. 360:1-351.

[15] Krommes, J. A. (1993). Dielectric response and thermal fluctuations in gyrokinetic plasma. Phys. Fluids B 5:1066-1100.

[16] Krommes, J. A., Lee, W. W., Oberman, C. (1986). Equilibrium fluctuation energy of gyrokinetic plasma. Phys. Fluids 29:2421-2425.

[17] Hasegawa, A., Mima, K. (1978). Pseudo-three-dimensional turbulence in magnetized nonuniform plasma. Phys. Fluids 21:87-92.

[18] Braginskii, S. I. (1965). Transport processes in a plasma. In: Leontovich, M. N., ed. Reviews of Plasma Physics, Vol. 1. New York: Consultants Bureau, pp. 205-311.

[19] Hammett, G. W., Perkins, F. W. (1990). Fluid moment models for Landau damping with application to the ion-temperature-gradient instability. Phys. Rev. Lett. 64:3019-3022.

[20] Hasegawa, A., Wakatani, M. (1983). Plasma edge turbulence. Phys. Rev. Lett. 50:682-686. 
[21] Hu, G., Krommes, J. A., Bowman, J. C. (1997). Statistical theory of resistive drift-wave turbulence and transport. Phys. Plasmas 4:2116-2133.

[22] Koniges, A. E., Craddock, G. G. (1994). Trends, transport, and self-organization in fluid models of plasma turbulence. In: Current Topics in the Physics of Fluids. Trivandrum, India: Research Trends, pp. 485-506.

[23] Birdsall, C. K., Langdon, A. B. (1985). Plasma Physics via Computer Simulation. New York: McGraw-Hill.

[24] Krommes, J. A., Rath, S. (2003). Monte Carlo sampling of negative-temperature states, with application to nonlinear gyrokinetic plasma simulations. Phys. Rev. E 67:066402 (1-28).

[25] Hu, G., Krommes, J. A. (1994). Generalized weighting scheme for $\delta f$ particle-simulation method. Phys. Plasmas 1:863-874.

[26] Krommes, J. A. (1993). Thermal fluctuations in gyrokinetic plasma at finite beta. Phys. Rev. Lett. 70:3067-3070.

[27] Krommes, J. A. (1992). Advances in gyrokinetic fluctuation theory. Bull. Am. Phys. Soc. $37: 1590$.

[28] Kraichnan, R. H. (1959). The structure of isotropic turbulence at very high Reynolds numbers. J. Fluid Mech. 5:497-543.

[29] Vedenov, A., Velikhov, E., Sagdeev, R. (1962). The quasi-linear theory of plasma oscillations. In: Proceedings of the Conference on Plasma Physics and Controlled Nuclear Fusion Research (Salzburg, 1961) [Nucl. Fusion Suppl. Pt. 2]. Vienna: International Atomic Energy Agency, pp. 465-475 [Translated in U.S.A.E.C. Division of Technical Information document AEC-tr-5589 (1963), pp. 204-237.].

[30] Drummond, W. E., Pines, D. (1962). Non-linear stability of plasma oscillations. In: Proceedings of the Conference on Plasma Physics and Controlled Nuclear Fusion Research (Salzburg, 1961) [Nucl. Fusion Suppl. Pt. 3]. Vienna: International Atomic Energy Agency, pp. 1049-1057.

[31] Davidson, R. C. (1972). Methods in Nonlinear Plasma Theory. New York: Academic Press.

[32] Sagdeev, R. Z., Galeev, A. A. (1969). Nonlinear Plasma Theory. New York: W. A. Benjamin.

[33] Dupree, T. H. (1966). A perturbation theory for strong plasma turbulence. Phys. Fluids 9:1773-1782.

[34] Dupree, T. H. (1967). Nonlinear theory of drift-wave turbulence and enhanced diffusion. 
Phys. Fluids 10:1049-1055.

[35] Orszag, S. A., Kraichnan, R. H. (1967). Model equations for strong turbulence in a Vlasov plasma. Phys. Fluids 10:1720-1736.

[36] Martin, P. C., Siggia, E. D., Rose, H. A. (1973). Statistical dynamics of classical systems. Phys. Rev. A 8:423-437.

[37] Krommes, J. A. (1978). Turbulence, clumps, and the Bethe-Salpeter equation. In: Theoretical and Computational Plasma Physics. Vienna: International Atomic Energy Agency, pp. 405417.

[38] DuBois, D. F., Espedal, M. (1978). Direct interaction approximation and plasma turbulence theory. Plasma Phys. 20:1209-1239.

[39] Krommes, J. A. (1982). The direct-interaction approximation and statistically steady states of three nonlinearly coupled modes. Phys. Fluids 25:1393-1395.

[40] Kraichnan, R. H. (1963). Direct-interaction approximation for a system of several interacting simple shear waves. Phys. Fluids 6:1603-1609.

[41] Orszag, S. A. (1977). Lectures on the statistical theory of turbulence. In: Balian, R., Peube, J.-L., eds. Fluid Dynamics. New York: Gordon and Breach, pp. 235-374.

[42] Bowman, J. C. (1992). Realizable Markovian Statistical Closures: General Theory and Application to Drift-wave Turbulence. PhD thesis: Princeton University.

[43] Bowman, J. C., Krommes, J. A., Ottaviani, M. (1993). The realizable Markovian closure. I. General theory, with application to three-wave dynamics. Phys. Fluids B 5:3558-3589.

[44] Hu, G., Krommes, J. A., Bowman, J. C. (1995). Resistive drift-wave plasma turbulence and the realizable Markovian closure. Phys. Lett. A 202:117-125.

[45] Bowman, J. C., Krommes, J. A. (1997). The realizable Markovian closure and realizable testfield model. II: Application to anisotropic drift-wave turbulence. Phys. Plasmas 4:3895-3909.

[46] Kraichnan, R. H. (1971). An almost-Markovian Galilean-invariant turbulence model. J. Fluid Mech. 47:513-524.

[47] Chen, H.-D., Chen, S., Kraichnan, R. H. (1989). Probability distribution of a stochastically advected scalar field. Phys. Rev. Lett. 63:2657-2660.

[48] Takaoka, M. (1999). Application of mapping closure to non-gaussian velocity fields. Phys. Fluids 11:2205-2214.

[49] Pratt, J., Krommes, J. A. (2002). Mapping closure for Hasegawa-Wakatani dynamics. Bull. 
Am. Phys. Soc. 47(9):198.

[50] Kraichnan, R. H. (1976). Eddy viscosity in two and three dimensions. J. Atmos. Sci. 33:15211536.

[51] Cowley, S. C., Kulsrud, R. M., Sudan, R. (1991). Considerations of ion-temperature-gradientdriven turbulence. Phys. Fluids B 3:2767-2782.

[52] Diamond, P. H., Rosenbluth, M. N., Hinton, F. L., Malkov, M., Fleischer, J., Smolyakov, A. (1998). Dynamics of zonal flows and self-regulating drift-wave turbulence. In: 17th IAEA Fusion Energy Conference. Vienna: International Atomic Energy Agency, pp. 14211428 (IAEA-CN-69/TH3/1).

[53] Smolyakov, A. I., Diamond, P. H. (1999). Generalized action invariants for drift waves-zonal flow systems. Phys. Plasmas 6:4410-4413.

[54] Krommes, J. A., Kim, C.-B. (2000). Interactions of disparate scales in drift-wave turbulence. Phys. Rev. E 62:8508-8539.

[55] Krommes, J. A., Kolesnikov, R. A. (2003). Hamiltonian description of zonal-flow generation. Phys. Plasmas (in press).

[56] Krommes, J. A. (2004). A roadmap to recent developments in the theory of turbulent generation of long-wavelength flows. J. Plasma Fusion Research (in press).

[57] Kolesnikov, R. A., Krommes, J. A. (2002). Bifurcation analysis of the Dimits upshift regime. Bull. Am. Phys. Soc. 47(9)(9):73.

[58] Rosenbluth, M. N., Hinton, F. L. (1998). Poloidal flow driven by ion-temperature-gradient turbulence in tokamaks. Phys. Rev. Lett. 80:724-727.

[59] Carnevale, G. F., Martin, P. C. (1982). Field theoretical techniques in statistical fluid dynamics: With application to nonlinear wave dynamics. Geophys. Astrophys. Fluid Dynamics 20:131-164.

[60] Martin, P. C. (1968). Measurements and correlation functions. In: deWitt, C., Balian, R., eds. Many Body Physics. New York: Gordon and Breach, pp. 37-136.

[61] Dyson, F. J. (1949). The radiation theories of Tomonaga, Schwinger, and Feynman. Phys. Rev. 75:486-502.

[62] Kim, E.-j., Diamond, P. H. (2002). Dynamics of zonal flow saturation in strong collisionless drift wave turbulence. Phys. Plasmas 9:4530-4539.

[63] Krommes, J. A. (2003). Comments on "Dynamics of zonal flow saturation in strong 
collisionless drift wave turbulence" [Phys. Plasmas 9, 4530 (2002)]. Phys. Plasmas (in press).

[64] Morrison, P. J., Greene, J. M. (1980). Noncanonical Hamiltonian density formulation of hydrodynamics and ideal magnetohydrodynamics. Phys. Rev. Lett. 45:790-794.

[65] Morrison, P. J. (1998). Hamiltonian description of the ideal fluid. Rev. Mod. Phys. 70:467-521.

[66] Ottaviani, M., Bowman, J. C., Krommes, J. A. (1991). Advances in the analytical theory of plasma turbulence and transport: Realizable Markovian statistical closures. Phys. Fluids B 3:2186-2191.

[67] Kolesnikov, R. A. PhD thesis, Princeton U. (in preparation). 


\section{Figures}

FIG. 1: (a) A typical tokamak showing nested flux surfaces. (b) Local coordinate system for studying microturbulence. The turbulence is of much shorter wavelength than the scale length of the background profile.

FIG. 2: Let the magnetic field $\boldsymbol{B}$ point out of the page. A net deficiency of ion gyrocenters produces an inward-pointing electric field $\boldsymbol{E}$; the resulting $\boldsymbol{E} \times \boldsymbol{B}$ drift produces counterclockwise motion, i.e., a positive vorticity [in agreement with Eqs. (6) and (7)].

FIG. 3: Integration domains for the evolution of the small $\boldsymbol{q}$ 's. Region C: $k \geq k_{\min }$. Region D: $k<k_{\min }, p \geq k_{\min }$. (From Ref. 54.)

FIG. 4: Casimir conservation restricts the dynamics to lie in a subspace of the energy "surface." 


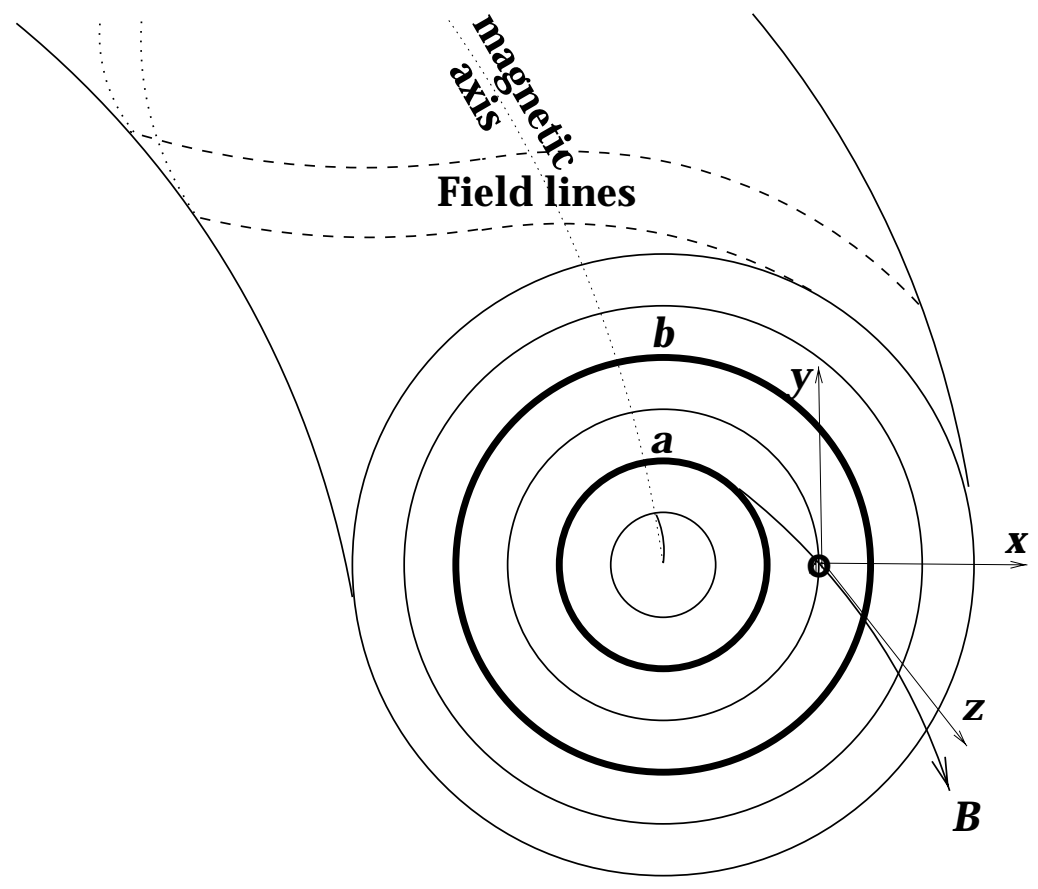

(a)

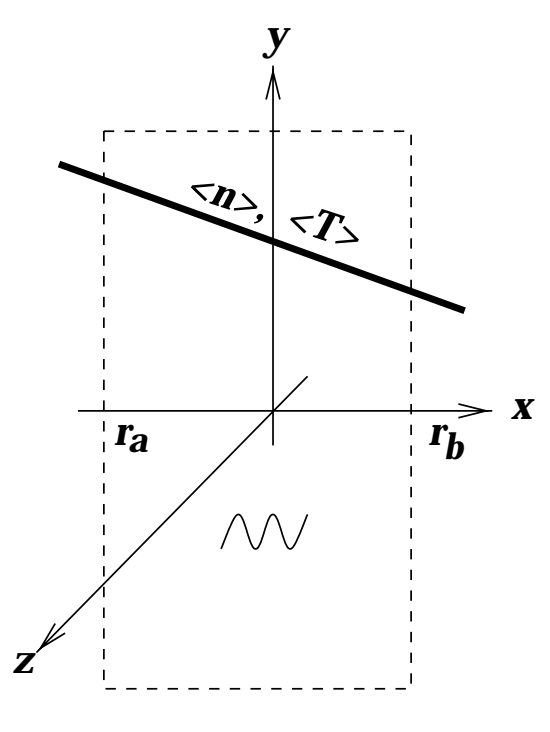

(b)

Fig. 1 


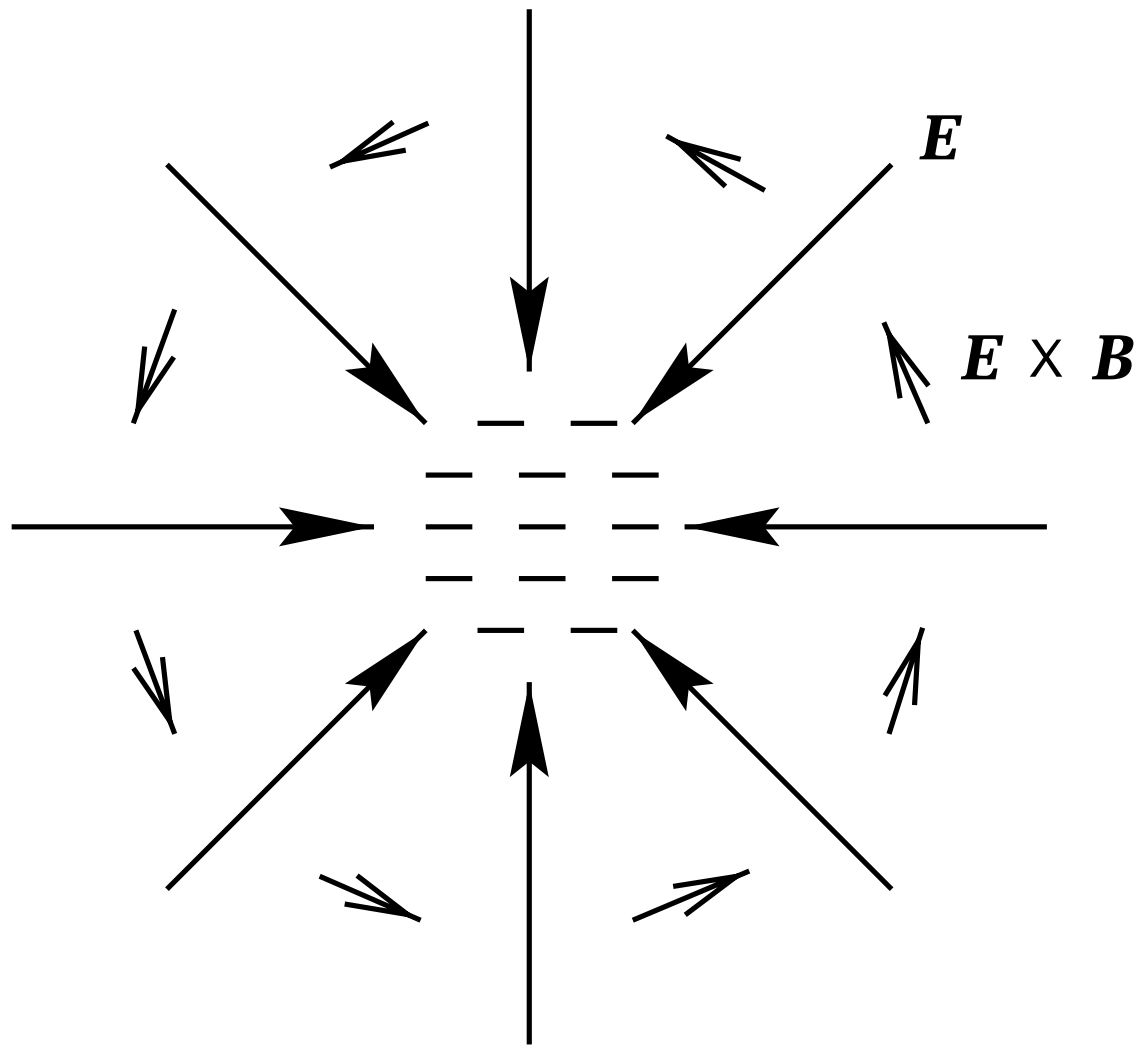

Fig. 2 


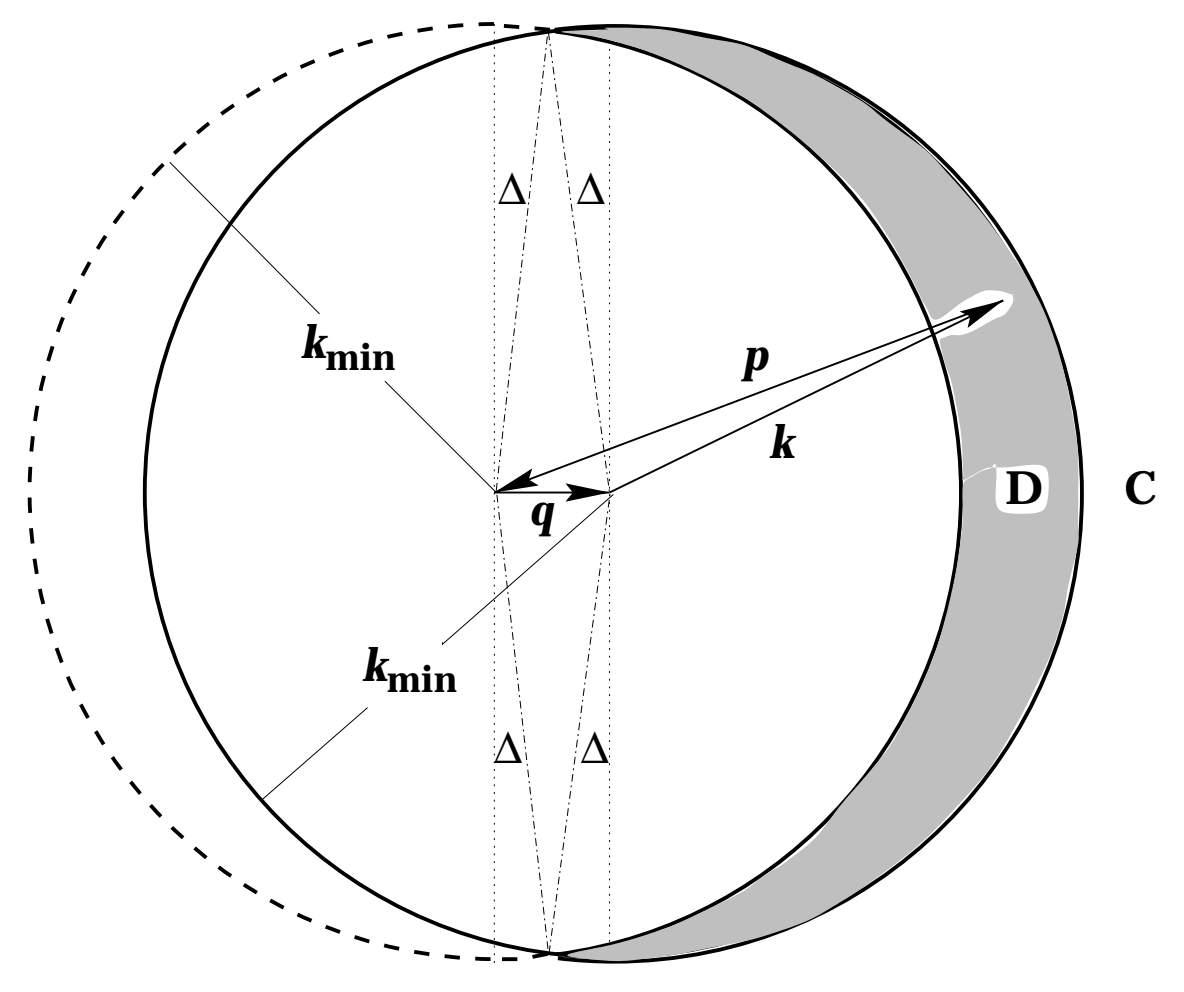

Fig. 3 


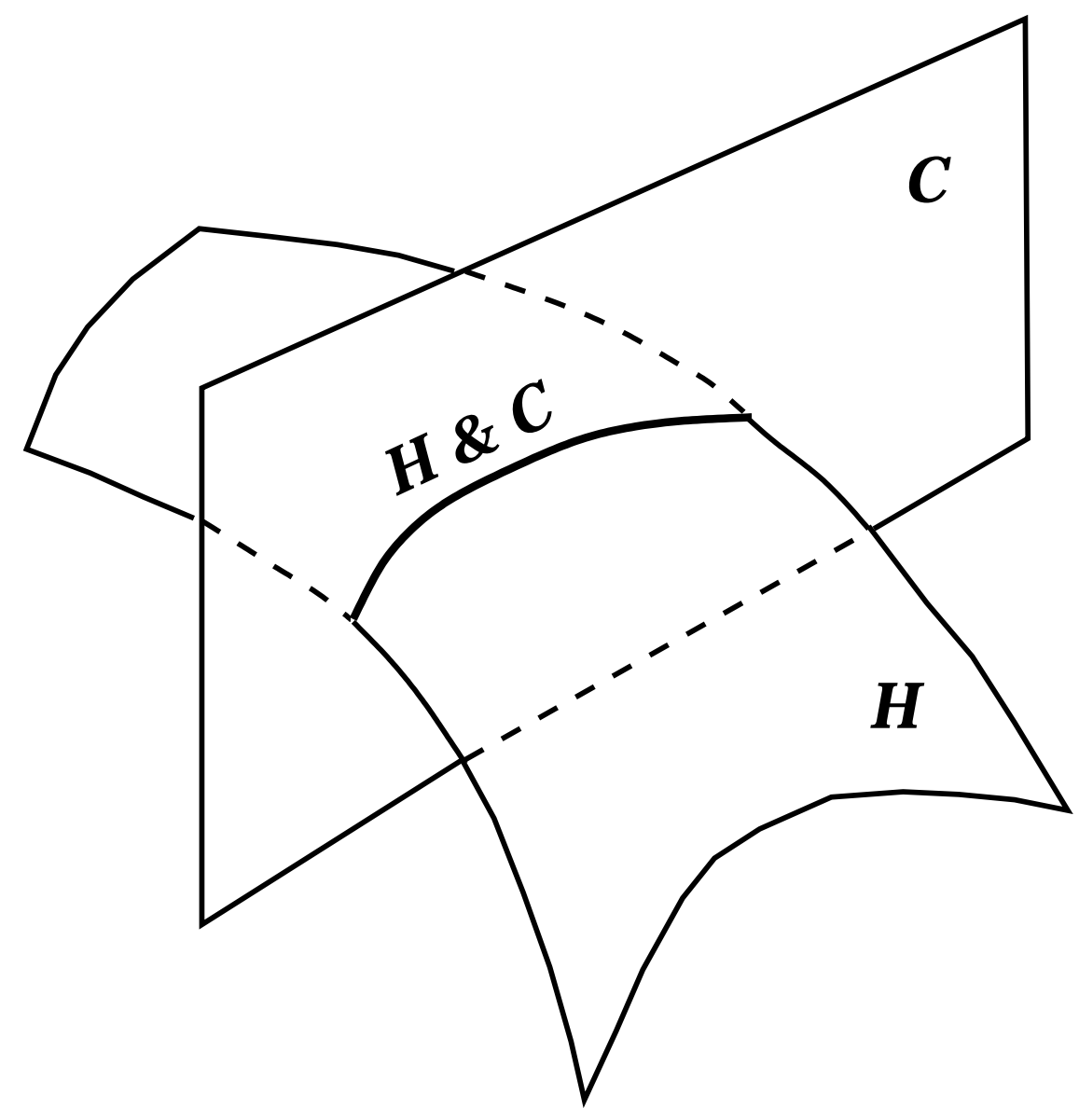

Fig. 4 


\section{External Distribution}

Plasma Research Laboratory, Australian National University, Australia

Professor I.R. Jones, Flinders University, Australia

Professor João Canalle, Instituto de Fisica DEQ/IF - UERJ, Brazil

Mr. Gerson O. Ludwig, Instituto Nacional de Pesquisas, Brazil

Dr. P.H. Sakanaka, Instituto Fisica, Brazil

The Librarian, Culham Laboratory, England

Mrs. S.A. Hutchinson, JET Library, England

Professor M.N. Bussac, Ecole Polytechnique, France

Librarian, Max-Planck-Institut für Plasmaphysik, Germany

Jolan Moldvai, Reports Library, Hungarian Academy of Sciences, Central Research Institute for Physics, Hungary

Dr. P. Kaw, Institute for Plasma Research, India

Ms. P.J. Pathak, Librarian, Institute for Plasma Research, India

Ms. Clelia De Palo, Associazione EURATOM-ENEA, Italy

Dr. G. Grosso, Instituto di Fisica del Plasma, Italy

Librarian, Naka Fusion Research Establishment, JAERI, Japan

Library, Laboratory for Complex Energy Processes, Institute for Advanced Study, Kyoto University, Japan

Research Information Center, National Institute for Fusion Science, Japan

Dr. O. Mitarai, Kyushu Tokai University, Japan

Dr. Jiangang Li, Institute of Plasma Physics, Chinese Academy of Sciences, People's Republic of China

Professor Yuping Huo, School of Physical Science and Technology, People's Republic of China

Library, Academia Sinica, Institute of Plasma Physics, People's Republic of China

Librarian, Institute of Physics, Chinese Academy of Sciences, People's Republic of China

Dr. S. Mirnov, TRINITI, Troitsk, Russian Federation, Russia

Dr. V.S. Strelkov, Kurchatov Institute, Russian Federation, Russia

Professor Peter Lukac, Katedra Fyziky Plazmy MFF UK, Mlynska dolina F-2, Komenskeho Univerzita, SK-842 15 Bratislava, Slovakia

Dr. G.S. Lee, Korea Basic Science Institute, South Korea

Institute for Plasma Research, University of Maryland, USA

Librarian, Fusion Energy Division, Oak Ridge National Laboratory, USA

Librarian, Institute of Fusion Studies, University of Texas, USA

Librarian, Magnetic Fusion Program, Lawrence Livermore National Laboratory, USA

Library, General Atomics, USA

Plasma Physics Group, Fusion Energy Research Program, University of California at San Diego, USA

Plasma Physics Library, Columbia University, USA

Alkesh Punjabi, Center for Fusion Research and Training, Hampton University, USA

Dr. W.M. Stacey, Fusion Research Center, Georgia Institute of Technology, USA

Dr. John Willis, U.S. Department of Energy, Office of Fusion Energy Sciences, USA

Mr. Paul H. Wright, Indianapolis, Indiana, USA 
The Princeton Plasma Physics Laboratory is operated by Princeton University under contract with the U.S. Department of Energy.

\author{
Information Services \\ Princeton Plasma Physics Laboratory \\ P.O. Box 451 \\ Princeton, NJ 08543
}

Phone: 609-243-2750

Fax: 609-243-2751

e-mail: pppl_info@pppl.gov

Internet Address: http://www.pppl.gov 